\title{
Full Discretization Scheme for the Dynamics of Elliptic Membrane Shell Model
}

\author{
Xiaoqin Shen ${ }^{1, *}$, Qian Yang ${ }^{1}$, Lin Bai ${ }^{1}$ and Kaitai $\mathrm{Li}^{2}$ \\ ${ }^{1}$ Department of Mathematics, School of Sciences, Xi'an University of Technology, \\ $X i^{\prime} a n, 710054$, Shaanxi, China. \\ 2 School of Mathematics and Statistics, Xi'an Jiaotong University, Xi'an, 710049, \\ Shaanxi, China.
}

Received 12 December 2019; Accepted (in revised version) 14 March 2020

\begin{abstract}
In this study, the dynamics of elliptic membrane shell model has been proposed and discussed numerically for the first time. Firstly, we show that the solution of this model exists and is unique. Secondly, we consider spatial and time discretizations of the time-dependent elliptic membrane shell by finite element method and Newmark scheme, respectively. Then, the corresponding existence, uniqueness, stability, convergence and a priori error estimate are given. Finally, we present numerical results involving a portion of an ellipsoidal shell and a portion of a spherical shell to verify the efficiency and convergence of the numerical scheme.
\end{abstract}

AMS subject classifications: 65N12, 65N15, 65N30

Key words: Elliptic membrane shell, finite element method, full discretization, ellipsoidal shell, spherical shell.

\section{Introduction}

Elliptic shells, such as ellipsoidal shells and spherical shells, have been applied in many fields, such as biological engineering (cf. [1,2]), composite material (cf. [3]), artificial intelligence (cf. [4]), the construction of nuclear power plants building (cf. [5]), magnetic industry (cf. $[6,7])$, and marine structures like egg-shaped pressure hull (cf. [8]). The theory of elliptic membrane shells is one of the most important branches in elastic shells theory.

In 1973, the theory of shells and plates was proposed in [9]. The mathematical justification for membrane shells model and generalized membrane shells model was given,

*Corresponding author. Email addresses: xqshen@xaut.edu.cn (X. Shen), yq931122@sina.com (Q. Yang), bailin931107@163.com (L. Bai), ktli@xjtu.edu.cn (K. Li) 
respectively, in [10] and [11]. The error estimates for the membrane shells model and the analysis of generalized membrane shell with hyperbolic [12] and parabolic [13] middle surfaces were given, respectively. Ciarlet [14] provided a detailed introduction on elliptic membrane shell including definitions, theories and examples. The elliptic membrane shell is defined by means of its middle surface, which is required to be uniformly elliptic and clamped along the entire boundary. Later, the Donati compatibility conditions for membrane shells through the intrinsic approach, i.e., a quadratic minimization problem with the linearized change of metric and change of curvature tensors of the middle surface of the shell as the new unknowns, were discussed in [15].

For the difficult problem of dynamic membrane shells, which can be equivalently formulated by means of a set of hyperbolic equations, the literature related to its theory or model is quite sparse. For viscoelastic elliptic membrane shell, there are also very few theoretical results. In [16], for instance, the authors justified the two-dimensional equations of a viscoelastic elliptic membrane shell, which includes a long-term memory that takes into account previous deformations. In [17], Dong et al. provided a multi-scale computational method for dynamic thermo-mechanical performance of heterogeneous shell structures.

To the best of our knowledge, there is rare literature [18] related to the numerical simulation of the time-dependent model involving membrane shells, which is based on the steady generalized membrane shell model proposed in [14]. The study of the more general time-dependent Koiter's model has been discussed in [19] and [20]. Compared with the generalized membrane shell, the main numerical difficulties for the elliptic membrane shell model is that the geometric hypothesis and boundary conditions are more strict.

In this paper, we study the time-dependent elliptic membrane shell model. Firstly, we established the time-dependent elliptic membrane shell model and proved the existence and uniqueness of the solution of the model. Secondly, the spatial variable is discretized by means of the finite element method and the full time discretization of the model is performed using the Newmark scheme. Then the corresponding results of existence, uniqueness, stability, convergence and a priori error estimate are given. These results rely on some technical approximation hypotheses for which we provide some references. Finally, we provided numerical experiments for a portion of an ellipsoidal shell and a portion of a spherical shell. In addition, the stability and validity of the model are verified by the calculation of the convergence order and error of the ellipsoid shells with different time steps and space steps.

\section{Preliminaries}

Our notation is essentially borrowed from [14]. In what follows, Latin indices and exponents take their values in the set $\{1,2,3\}$, whereas Greek indices and exponents take their values in the set $\{1,2\}$. In addition, the repeated index summation convention is systematically used. The Euclidean inner product and the exterior product of any vectors 
$\vec{a}, \vec{b} \in \mathbb{R}^{3}$ are denoted by $\vec{a} \cdot \vec{b}$ and $\vec{a} \times \vec{b}$, respectively.

Let $\omega$ be a domain in $\mathbb{R}^{2}$, in the sense of Section 1.18 of [21], with boundary $\gamma_{0}=\partial \omega$. Let $y=\left(y_{\alpha}\right)$ denote a generic point in the closure of the set $\omega$ and let $\partial_{\alpha}:=\partial / \partial y_{\alpha}$. Let $\vec{\theta} \in C^{3}\left(\bar{\omega} ; \mathbb{R}^{3}\right)$ be an injective mapping such that the two vectors (cf. [22])

$$
\vec{a}_{\alpha}(y):=\partial_{\alpha} \vec{\theta}(y), \quad \alpha=1,2,
$$

are linearly independent at all points $y \in \bar{\omega}$. These two vectors thus span the tangent plane to the surface

$$
S:=\vec{\theta}(\bar{\omega}),
$$

at the point $\vec{\theta}(y)$, and the unit vector

$$
\vec{a}_{3}(y):=\frac{\vec{a}_{1}(y) \times \vec{a}_{2}(y)}{\left|\vec{a}_{1}(y) \times \vec{a}_{2}(y)\right|}
$$

is normal to the tangent plane to the surface $S$ at the point $\vec{\theta}(y)$. The vectors $\vec{a}_{i}(y)$ constitute the covariant basis at the point $\vec{\theta}(y)$, whereas the vectors $\vec{a}^{i}(y)$, defined by the relations

$$
\vec{a}^{i}(y) \cdot \vec{a}_{j}(y)=\delta_{j}^{i}
$$

constitute the contravariant basis at the point $\vec{\theta}(y)$, where $\delta_{j}^{i}$ is the Kronecker symbol (note that $\vec{a}^{3}(y)=\vec{a}_{3}(y)$ and that the vectors $\vec{a}^{\alpha}(y)$ span the tangent plane to $S$ at the point $\vec{\theta}(y))$.

We can define the first fundamental form, as a metric tensor, in covariant or contravariant components, respectively, by

$$
a_{\alpha \beta}:=\vec{a}_{\alpha} \cdot \vec{a}_{\beta}, \quad a^{\alpha \beta}:=\vec{a}^{\alpha} \cdot \vec{a}^{\beta},
$$

the second fundamental form, as a curvature tensor, in covariant or mixed components, respectively, by

$$
b_{\alpha \beta}:=\vec{a}^{3} \cdot \partial_{\beta} \vec{a}_{\alpha}, \quad b_{\alpha}^{\beta}:=a^{\beta \sigma} \cdot b_{\sigma \alpha},
$$

as well as the Christoffel symbols of the surface $S$ as

$$
\Gamma_{\alpha \beta}^{\sigma}:=\vec{a}^{\sigma} \cdot \partial_{\beta} \vec{a}_{\alpha} .
$$

The area element along $S$ is $\sqrt{a} d y$, where $a:=\operatorname{det}\left(a_{\alpha \beta}\right)$.

The covariant derivatives on $S$ are defined as follows:

$$
\begin{aligned}
& \eta_{\alpha \mid \beta}:=\partial_{\beta} \eta_{\alpha}-\Gamma_{\alpha \beta}^{\sigma} \eta_{\sigma}, \quad \eta_{3 \mid \beta}:=\partial_{\beta} \eta_{3}, \\
& \left.\eta^{\alpha}\right|_{\beta}:=\partial_{\beta} \eta^{\alpha}+\Gamma_{\beta \sigma}^{\alpha} \eta^{\sigma},\left.\quad \eta^{3}\right|_{\beta}:=\partial_{\beta} \eta^{3}, \\
& \left.\eta^{\alpha \beta}\right|_{\sigma}:=\partial_{\sigma} \eta^{\alpha \beta}+\Gamma_{\sigma \tau}^{\alpha} \eta^{\beta \tau}+\Gamma_{\sigma \tau}^{\beta} \eta^{\alpha \tau}, \\
& \left.b_{\alpha}^{\beta}\right|_{\sigma}:=\partial_{\sigma} b_{\alpha}^{\beta}+\Gamma_{\sigma \tau}^{\beta} b_{\alpha}^{\tau}-\Gamma_{\alpha \sigma}^{\tau} b_{\tau}^{\beta},
\end{aligned}
$$


where $\vec{\eta}$ is the displacement of the middle surface of the shell.

We introduce the fourth-order two-dimensional elasticity tensor on $S$ by means of its contravariant components $a^{\alpha \beta \sigma \tau}$, defined by

$$
a^{\alpha \beta \sigma \tau}:=\frac{4 \lambda \mu}{\lambda+2 \mu} a^{\alpha \beta} a^{\sigma \tau}+2 \mu\left(a^{\alpha \sigma} a^{\beta \tau}+a^{\alpha \tau} a^{\beta \sigma}\right),
$$

where $\lambda \geq 0, \mu>0$ are the Lamé constants associated with the constituting material.

\section{Dynamics of elliptic membrane shell}

Assume that we are given a shell with middle surface $S=\vec{\theta}(\bar{\omega})$ where the mapping $\vec{\theta} \in$ $C^{3}\left(\bar{\omega} ; \mathbb{R}^{3}\right)$ is such that the two vectors $\vec{a}_{\alpha}=\partial_{\alpha} \vec{\theta}(y)$ are linearly independent at all points $y \in \bar{\omega}$, and whose thickness $2 \varepsilon>0$ approaches zero (cf. Fig. 1).

Hence, for each $\varepsilon>0$, the reference configuration of the shell is $\vec{\Theta}(\bar{\Omega})$, where $\bar{\Omega}=$ $\bar{\omega} \times[-\varepsilon, \varepsilon]$, i.e.,

$$
\vec{\Theta}(y, \xi)=\vec{\theta}(y)+\xi \vec{a}_{3}(y), \quad \text { for all } \quad(y, \xi) \in \bar{\omega} \times[-\varepsilon, \varepsilon] \text {. }
$$

Note that the mapping $\vec{\Theta}: \bar{\Omega} \rightarrow \mathbb{R}^{3}$ is such that $\nabla \vec{\Theta}(y, \xi)$ is an invertible matrix at all points $(y, \xi) \in \bar{\omega} \times[-\varepsilon, \varepsilon]$, provided that $\varepsilon$ is small enough (cf. Theorem 3.1-1 of [14]).

Let $\gamma_{\alpha \beta}$ denote the covariant components of the change of metric tensor, which are defined as follows

$$
\begin{aligned}
\gamma_{\alpha \beta}(\vec{\eta}):= & \frac{1}{2}\left(\partial_{\alpha} \eta_{\beta}+\partial_{\beta} \eta_{\alpha}\right)-\Gamma_{\alpha \beta}^{\sigma} \eta_{\sigma}-b_{\alpha \beta} \eta_{3}, \\
& \text { for all } \vec{\eta} \in H^{1}(\omega) \times H^{1}(\omega) \times L^{2}(\omega) .
\end{aligned}
$$

Following [14], let us recall the definition of membrane shell. A linearly elastic shell with middle surface $S=\vec{\theta}(\bar{\omega})$ is called an elliptic membrane shell if the following conditions are simultaneously satisfied:

(i) The shell is subject to a boundary condition of place along its entire lateral face $\vec{\Theta}\left(\gamma_{0}\right)$ as its middle curve;

(ii) The middle surface $S$ is elliptic, i.e., the Gaussian curvature of $S$ is everywhere strictly positive. Equivalently, the two principal radii of curvature are either both positive at all points of $S$, or both negative at all points of $S$.

Let us introduce the space

$$
\vec{V}_{M}(\omega):=\left\{\vec{\eta}=\left(\eta_{i}\right) \in H_{0}^{1}(\omega) \times H_{0}^{1}(\omega) \times L^{2}(\omega)\right\},
$$

and the space

$$
\vec{H}:=\vec{L}^{2}(\omega)
$$




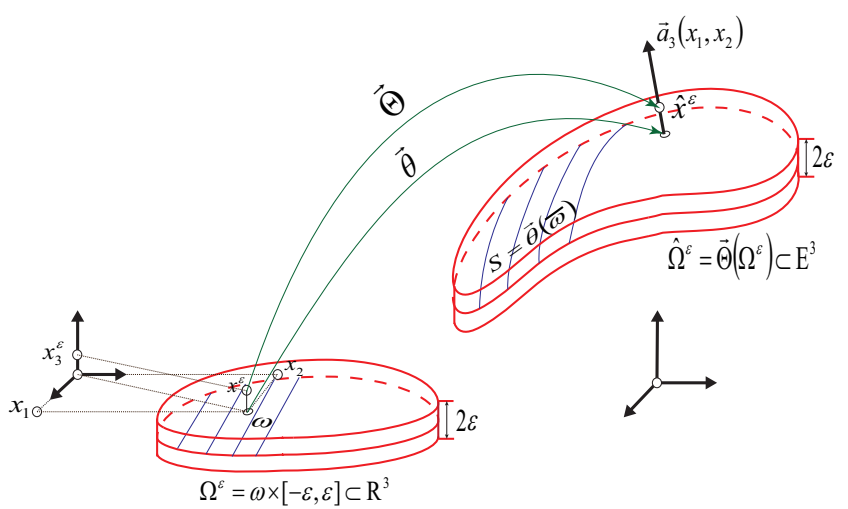

(a)

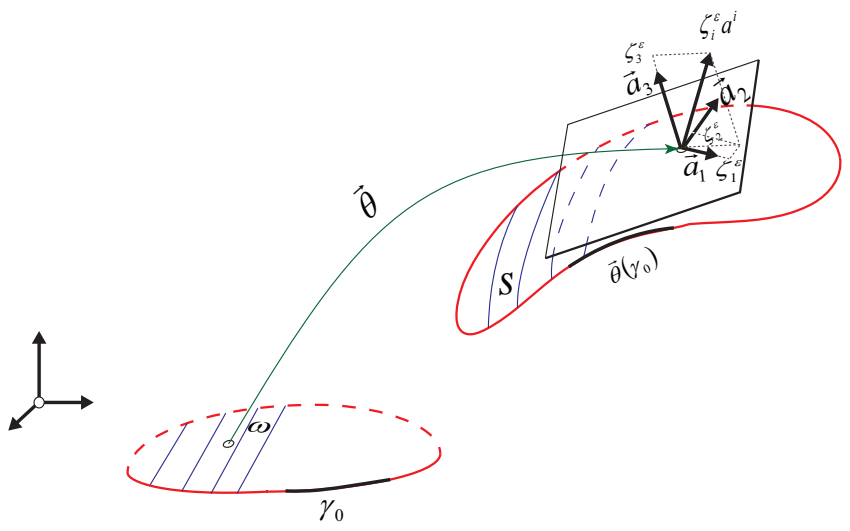

(b)

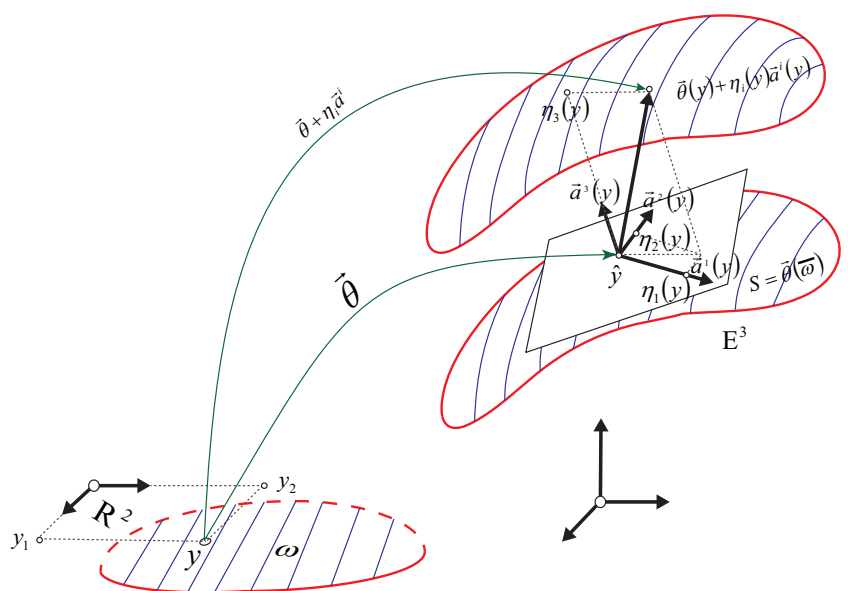

(c)

Figure 1: Shell kinematics (cf. [14]): (a) Three-dimensional shell $\hat{\Omega}^{\varepsilon}=\vec{\Theta}(\bar{\Omega})$ with middle surface $S=\vec{\theta}(\bar{\omega}) ;($ b) two-dimensional shell $S=\vec{\theta}(\bar{\omega})$; (c) contravariant basis and displacement vector filed along $S$. 
In what follows we investigate the evolution of the displacement of the studied membrane shell in the time interval $[0, T]$, where $T>0$ is a given constant. We study the corresponding two dimensional scaled model (cf. [14]) and, therefore, in what follows we denote $\left(y, x_{3}\right)$ the generic point of the set $\bar{\omega} \times[-1,1]$. Let us state the main problem.

Problem 1. Finding a vector field $\vec{\zeta}:[0, T] \rightarrow \vec{V}_{M}(\omega)$ satisfying the following variational equations

$$
2 \varepsilon \rho \int_{\omega} \frac{d^{2}}{d t^{2}} \zeta^{i}(t) \eta_{i} \sqrt{a} d y+\varepsilon \int_{\omega} a^{\alpha \beta \sigma \tau} \gamma_{\sigma \tau}(\vec{\zeta}(t)) \gamma_{\alpha \beta}(\vec{\eta}) \sqrt{a} d y=\int_{\omega} p^{i, \varepsilon}(t) \eta_{i} \sqrt{a} d y,
$$

for all $\vec{\eta} \in \vec{V}_{M}(\omega)$, in the sense of distributions in $(0, T)$, as well as the following initial conditions at $t=0$

$$
\begin{aligned}
& \vec{\zeta}(0)=\vec{\zeta}_{0}, \\
& \frac{d}{d t} \vec{\zeta}(0)=\vec{\zeta}_{1},
\end{aligned}
$$

where $\rho$ is the mass density of the constitutive material, which is assumed to be homogeneous and isotropic, the thickness of the shell is $2 \varepsilon$,

$$
\zeta^{\alpha}(t):=a^{\alpha \beta} \zeta_{\beta}(t), \quad \zeta^{3}(t):=\zeta_{3}(t),
$$

and the vectors $\vec{\zeta}_{0} \in \vec{V}_{M}(\omega)$ and $\vec{\zeta}_{1} \in \vec{H}$ are given. The functions $p^{i, \varepsilon}(t)$ denote the contravariant components of the applied forces (this means that $p^{i, \varepsilon}(t, y) \vec{a}_{i}(y) \sqrt{a(y)} d y$ is the force acting on the area element $\sqrt{a(y)} d y$ of $S$ at the time $t)$. For a.a. $t \in(0, T)$, thanks to the main identification property of Lebesgue-Bochner spaces, we can define each component $p^{i, \varepsilon}(t)$ as

$$
p^{i, \varepsilon}(\cdot, t):=\varepsilon\left\{\int_{-1}^{1} f^{i}(\cdot, t) d x_{3}+h_{+}^{i}(\cdot, t)+h_{-}^{i}(\cdot, t)\right\},
$$

where $f^{i} \in L^{2}\left(0, T ; L^{2}(\omega \times(-1,1))\right)$ denotes the given applied body force density and, for a.a. $t \in(0, T), h_{+}^{i}(\cdot, t) \in L^{2}(\omega \times\{1\})$ and $h_{-}^{i}(\cdot, t) \in L^{2}(\omega \times\{-1\})$ denote the given applied surface force densities.

As a result, the to-be-sought unknown is the vector field $\vec{\zeta}(t)=\left(\zeta_{i}(t)\right): \bar{\omega} \rightarrow \mathbb{R}^{3}$. For each $y \in \bar{\omega}$ and each $t \geq 0, \zeta_{i}(t, y) \vec{a}^{i}(y)$ represents the displacement vector field of the point $\vec{\theta}(y) \in S$ at the time $t$.

Let us define the semi-norm $|\cdot|_{\omega}^{M}$ as (cf. [14]),

$$
|\vec{\eta}|_{\omega}^{M}:=\left\{\sum_{\alpha, \beta}\left|\gamma_{\alpha \beta}(\vec{\eta})\right|_{0, \omega}^{2}\right\}^{1 / 2},
$$

for all $\vec{\eta}=\left(\eta_{i}\right) \in H^{1}(\omega) \times H^{1}(\omega) \times L^{2}(\omega)$, which is equivalent to the norm $\|\cdot\|_{\vec{V}_{M}(\omega)}$, according to an inequality of Korn's type on an elliptic surface (cf. Theorem 2.7-3 of [14]). 
For sake of brevity, let us define, using the same notation as in Section 8.2 of [23],

$$
\begin{aligned}
(\vec{\zeta}(t), \vec{\eta}) & :=2 \varepsilon \rho \int_{\omega} \zeta^{i}(t) \eta_{i} \sqrt{a} d y, \\
B(\vec{\zeta}(t), \vec{\eta}) & :=\varepsilon \int_{\omega} a^{\alpha \beta \sigma \tau} \gamma_{\sigma \tau}(\vec{\zeta}(t)) \gamma_{\alpha \beta}(\vec{\eta}) \sqrt{a} d y, \\
(\vec{p}(t), \vec{\eta}) & :=\int_{\omega} p^{i, \varepsilon}(t) \eta_{i} \sqrt{a} d y,
\end{aligned}
$$

where $\vec{\eta} \in \vec{V}_{M}(\omega)$.

Let us state Problem 1 in a more condensed form.

Problem 2. For each $t \in(0, T]$ find $\vec{\zeta}(t) \in \vec{V}_{M}(\omega)$ that satisfies

$$
\frac{d^{2}}{d t^{2}}(\vec{\zeta}(t), \vec{\eta})+B(\vec{\zeta}(t), \vec{\eta})=(\vec{p}(t), \vec{\eta})
$$

for all $\vec{\eta} \in \vec{V}_{M}(\omega)$, in the sense of distributions in $(0, T)$, as well as the following initial conditions at $t=0$

$$
\vec{\zeta}(0)=\vec{\zeta}_{0}, \quad \frac{d \vec{\zeta}}{d t}(0)=\vec{\zeta}_{1}
$$

where $\vec{\zeta}_{0} \in \vec{V}_{M}(\omega)$ and $\vec{\zeta}_{1} \in \vec{H}$ are given.

Theorem 3.1. Assume that $\vec{p}=\left(p^{i, \varepsilon}\right) \in L^{2}(0, T ; \vec{H})$. Then, Problem 2 (and so Problem 1 ) has one and only one solution $\vec{\zeta} \in C^{0}\left(0, T ; \vec{V}_{M}(\omega)\right) \cap C^{1}(0, T ; \vec{H})$.

Proof. The space $\vec{V}_{M}(\omega)$ is contained in the space $\vec{H}$ with a compact canonical injection and $\vec{V}_{M}(\omega)$ is dense in $\vec{H}$. The bilinear form $B(\cdot, \cdot)$, which is clearly symmetric and continuous, is also $\vec{V}_{M}(\omega)$-elliptic (cf. [10]). By virtue of Theorem 8.2-2 of [23] (cf. also [24] and [25]), we can thus deduce that Problem 2 has one and only one solution $\vec{\zeta} \in C^{0}\left(0, T ; \vec{V}_{M}(\omega)\right) \cap C^{1}(0, T ; \vec{H})$.

\section{Numerical methods for approximating the solution: Semi-discretization in space}

The spatial variable is discretized by means of the finite element method as proposed in $[18-20,26,27]$, i.e., given a regular triangulation $\tau_{h}$ (in the sense of [26]) of the domain $\bar{\omega}$ (henceforth assumed to be polygonal) made of triangles $\mathbb{K} \in \tau_{h}$, we use a P1b conforming finite element (piecewise linear continuous plus bubble element, see [28]) for approximating the first two components $\zeta_{\alpha}$ of the unknown displacement whereas we use a PO conforming finite element (cf. [28]) for the third component. More specifically, we let

$$
\vec{V}_{h}:=V_{h, 1} \times V_{h, 2} \times V_{h, 3}
$$


where

$$
\begin{aligned}
& V_{h, \alpha}:=\left\{\eta_{h} \in C^{0}(\bar{\omega}) ;\left.\eta_{h}\right|_{\mathbb{K}} \in P_{1}(\mathbb{K}) \oplus \operatorname{Span}\left\{\lambda_{0}^{\mathbb{K}} \lambda_{1}^{\mathbb{K}} \lambda_{2}^{\mathbb{K}}\right\}, \text { for each } \mathbb{K} \in \tau_{h} \text { and } \eta_{h}=0 \text { on } \gamma_{0}\right\}, \\
& V_{h, 3}:=\left\{\eta_{h} \in L^{2}(\omega) ;\left.\eta_{h}\right|_{\mathbb{K}} \text { is constant, for each } \mathbb{K} \in \tau_{h}\right\},
\end{aligned}
$$

where the notation $P_{k}(\mathbb{K}), k \geq 1$, designates the space formed by polynomials in two variables of degree less than or equal to $k$ restricted to a triangle $\mathbb{K} \in \tau_{h}$.

The approximation of Problem 3 takes the following form.

Problem 3. For each $t \in(0, T]$, finding a vector field $\vec{\zeta}_{h}(t) \in \vec{V}_{h}$ that satisfies

$$
\frac{d^{2}}{d t^{2}}\left(\vec{\zeta}_{h}(t), \vec{\eta}_{h}\right)+B\left(\vec{\zeta}_{h}(t), \vec{\eta}_{h}\right)=\left(\vec{p}(t), \vec{\eta}_{h}\right)
$$

for all $\vec{\eta}_{h} \in \vec{V}_{h}$ and for which the following initial conditions hold

$$
\vec{\zeta}_{h}(0)=\vec{\zeta}_{0, h}, \quad \frac{d \vec{\zeta}_{h}}{d t}(0)=\vec{\zeta}_{1, h}
$$

where $\vec{\zeta}_{0, h}$ is the projection of the given vector field $\vec{\zeta}_{0}$ onto the space $\vec{V}_{h}$ with respect to the $\vec{L}^{2}(\omega)$ inner product, i.e.,

$$
\vec{\zeta}_{0, h}=\sum_{k=1}^{m}\left(\vec{\zeta}_{0}, \vec{w}_{k}\right)_{\vec{L}^{2}(\omega)} \vec{w}_{k}
$$

where $m=\operatorname{dim} \vec{V}_{h},\left(\vec{w}_{k}\right)_{k=1}^{m}$ is an orthogonal basis in $\vec{V}_{M}(\omega)$ which is also a Hilbert basis in $\vec{L}^{2}(\omega)$ (see Theorem 6.2-1 of [23]). Likewise, $\vec{\zeta}_{1, h}$ is the projection of the given vector field $\vec{\zeta}_{1}$ onto the space $\vec{V}_{h}$ with respect to the $\vec{L}^{2}(\omega)$ inner product, i.e.,

$$
\vec{\zeta}_{1, h}=\sum_{k=1}^{m}\left(\vec{\zeta}_{1}, \vec{w}_{k}\right)_{\vec{L}^{2}(\omega)} \vec{w}_{k}
$$

where $m=\operatorname{dim} \vec{V}_{h},\left(\vec{w}_{k}\right)_{k=1}^{m}$ is an orthogonal basis in $\vec{V}_{M}(\omega)$ which is also a Hilbert basis in $\vec{L}^{2}(\omega)$ (see Theorem $6.2-1$ of [23]).

The proof of the following results comes straightforwardly (cf. Theorem 8.4-1 in [23] or Section 14.5 in [24]):

Theorem 4.1. Let the assumptions be the same as in Theorem 3.1. Then Problem 3 has one and only one solution.

The advantage of using a middle surface defined by a specific mapping $\vec{\theta}$ is that all the coefficients appearing in the integrals defining the inner product of the space $\vec{H},(\cdot, \cdot)$, and the bilinear form $B(\cdot, \cdot)$ can be computed exactly (in contrast with the case where the 
middle surface is only known at a finite number of points and possibly of tangent planes at this point; see, e.g., [26]).

Using the classical interpolation properties of the linear triangle element (cf. [26]), we then obtain the following result (cf. Corollary 8.4-1 of [23] and also Theorem 8.7-1 of [24]) which makes a technical approximation assumption that we do not explicitly state but that, however, is worth mentioning for sake of completeness.

Theorem 4.2. Assume that $\vec{\zeta} \in C^{2}\left(0, T ; \vec{V}_{M}(\omega)\right)$ and that the approximation hypothesis (8.4-8) of [23] and

$$
\lim _{h \rightarrow 0}\left\|\vec{\zeta}_{0, h}-\vec{\zeta}_{0}\right\|_{\vec{V}_{M}(\omega)}=0, \quad \lim _{h \rightarrow 0}\left|\vec{\zeta}_{1, h}-\vec{\zeta}_{1}\right|_{\vec{H}}=0,
$$

hold, where $h:=\max _{T \in \tau_{h}} \operatorname{diam} T$ and diam $T$ is the diameter of $T$ in the sense of [26].

Then, it results

$$
\lim _{h \rightarrow 0}\left\{\left\|\vec{\zeta}_{h}(t)-\vec{\zeta}(t)\right\|_{\vec{V}_{M}(\omega)}+\left|\frac{d}{d t}\left(\vec{\zeta}_{h}(t)-\vec{\zeta}(t)\right)\right|_{\vec{H}}\right\}=0, \quad \text { for each } \quad 0 \leq t \leq T .
$$

\section{Numerical methods for approximating the solution: Full discretization}

The time discretization of Problem 3 is performed using the Newmark scheme (cf. [23, $24,29,30]$ ): Given a positive integer $N$, we introduce the time step $\Delta t=T / N$ and the discrete time $t_{n}=n \triangle t$, where $n=0, \cdots, N$. Let us introduce the approximation $\vec{\zeta}_{h}^{n} \approx \vec{\zeta}_{h}\left(t_{n}\right)$ and $\vec{p}^{n}:=\vec{p}\left(t_{n}\right)$, where $n=0, \cdots, N$.

Then, we can state the following full space-time discretization scheme for Problem 3: Finding $\vec{\zeta}_{h}^{n}$ such that

$$
\begin{aligned}
& \frac{1}{\triangle t^{2}}\left(\vec{\zeta}_{h}^{n+2}-2 \vec{\zeta}_{h}^{n+1}+\vec{\zeta}_{h}^{n}, \vec{\eta}_{h}\right)+B\left(\beta \vec{\zeta}_{h}^{n+2}+\left(\frac{1}{2}-2 \beta+\gamma\right) \vec{\zeta}_{h}^{n+1}+\left(\frac{1}{2}+\beta-\gamma\right) \vec{\zeta}_{h}^{n}, \vec{\eta}_{h}\right) \\
& \quad=\left(\beta \vec{p}^{n+2}+\left(\frac{1}{2}-2 \beta+\gamma\right) \vec{p}^{n+1}+\left(\frac{1}{2}+\beta-\gamma\right) \vec{p}^{n}, \vec{\eta}_{h}\right), \quad \forall \vec{\eta}_{h} \in \vec{V}_{h}, \quad n=0, \cdots, N-2, \\
& \vec{\zeta}_{h}^{0}:=\vec{\zeta}_{0, h}, \quad \frac{d \vec{\zeta}_{h}}{d t}(0)=\vec{\zeta}_{1, h \prime}
\end{aligned}
$$

where $\beta, \gamma \in \mathbb{R}$ are given parameters and $\vec{\zeta}_{h}^{1}$ is obtained by solving the following variational equations:

$$
\begin{aligned}
& \frac{1}{\triangle t^{2}}\left(\vec{\zeta}_{h}^{1}-\vec{\zeta}_{0, h}-\triangle t \vec{\zeta}_{1, h}, \vec{\eta}_{h}\right)+B\left(\beta \vec{\zeta}_{h}^{1}+\left(\frac{1}{2}-\beta\right) \vec{\zeta}_{0, h}, \vec{\eta}_{h}\right) \\
= & \left(\beta \vec{p}^{1}+\left(\frac{1}{2}-\beta\right) \vec{p}^{0}, \vec{\eta}_{h}\right), \quad \forall \vec{\eta}_{h} \in \vec{V}_{h} .
\end{aligned}
$$


In what follows, $\hat{\lambda}_{h}$ denotes the maximum eigenvalue recovered via the Spectral Theorem in the finite dimensional space $\vec{V}_{h}$. We now state the result concerning the existence, convergence and error estimate for the full space-time discretization scheme of the timedependent membrane shell model (cf. Theorem 8.7-2, Remark 8.7-3 of [23] and page 501 of [24]). The following result makes a technical approximation hypothesis that we do not explicitly state but that, however, is worth mentioning for sake of completeness.

Theorem 5.1 (Existence, uniqueness and convergence of the fully discrete solution). Let the assumptions be the same as in Theorem 3.1 and assume that the approximation hypothesis (6.5-2) of [23] holds. Let us also assume that

$$
\gamma>\frac{1}{2}, \quad \text { and } \quad \beta \geq \frac{(1 / 2+\gamma)^{2}}{4} .
$$

Then, the scheme (5.1) has one and only one solution $\vec{\zeta}_{h}^{n} \in \vec{V}_{h}, n=0, \cdots, N$.

Besides, if there exists a constant $\sigma>0$, independent of $h$ and $\triangle t$ but, eventually, depending on $\gamma$ and $\beta$, such that, if the stability condition

$$
\hat{\lambda}_{h} \Delta t^{2} \leq \sigma
$$

and if $\vec{\zeta}$ is smooth enough, then, there exists a constant $C>0$ independent of $h, \triangle t$ and $\vec{\zeta}$ but, eventually, dependent on $\sigma$, such that

$$
\left\|\vec{\zeta}_{h}^{n}-\vec{\zeta}\left(t_{n}\right)\right\|_{\vec{H}} \leq C\left\{\left\|\vec{\zeta}_{0, h}-\vec{\zeta}_{0}\right\|_{\vec{H}}+\left\|\vec{\zeta}_{1, h}-\vec{\zeta}_{1}\right\|_{\vec{H}}+h+\triangle t\right\}
$$

for all $n=0, \cdots, N$.

Proof. Under the assumptions of Theorem 8.7-2 and Remark 8.3-3 (cf. [23]), if $\vec{\zeta} \in$ $C^{2}\left(0, T ; \vec{H}^{l+1}(\omega)\right) \cap C^{3}\left(0, T ; \vec{L}^{2}(\omega)\right)$, and $\gamma>1 / 2$, the following estimates can be obtained:

$$
\left\|\zeta_{\alpha h}^{n}-\zeta_{\alpha}\left(t_{n}\right)\right\|_{L^{2}(\omega)} \leq C\left\{\left\|\zeta_{0, \alpha h}-\zeta_{0 \alpha}\right\|_{L^{2}(\omega)}+\left\|\zeta_{1, \alpha h}-\zeta_{1 \alpha}\right\|_{L^{2}(\omega)}+h^{k+1}+\triangle t\right\}, \quad \alpha=1,2,
$$

where $k=1$; and

$$
\left\|\zeta_{3 h}^{n}-\zeta_{3}\left(t_{n}\right)\right\|_{L^{2}(\omega)} \leq C\left\{\left\|\zeta_{0,3 h}-\zeta_{03}\right\|_{L^{2}(\omega)}+\left\|\zeta_{1,3 h}-\zeta_{13}\right\|_{L^{2}(\omega)}+h^{k+1}+\triangle t\right\},
$$

where $k=0$.

Thus, from $h^{2} \leq h$ ( $h$ is small enough), we can deduce that

$$
\left\|\vec{\zeta}_{h}^{n}-\vec{\zeta}\left(t_{n}\right)\right\|_{\vec{L}^{2}(\omega)} \leq C\left\{\left\|\vec{\zeta}_{0, h}-\vec{\zeta}_{0}\right\|_{\vec{L}^{2}(\omega)}+\left\|\vec{\zeta}_{1, h}-\vec{\zeta}_{1}\right\|_{\vec{L}^{2}(\omega)}+h+\triangle t\right\} .
$$

Based on the definition of $\vec{H}:=\vec{L}^{2}(\omega)$, then the error estimates can be derived directly. 
Remark 5.1. The condition $\gamma \geq 1 / 2$ is necessary for stability. If $\gamma=1 / 2$, it can be found that the convergence rate of Newmark scheme is second order with respect to time step (cf. [24]). Moreover, when $\gamma=1 / 2$ and $\beta=1 / 4$, the Newmark scheme is unconditionally stable. This popular choice is, however, unsuitable for long time integration, as the discrete solution may be affected by parasitic oscillations that are not damped as far as $t$ increases. For long time integration it is therefore preferable to choose $\beta \geq \frac{(1 / 2+\gamma)^{2}}{4}$ for a suitable $\gamma>1 / 2$, although in such a case the method degenerates to a first order with respect to time step.

\section{Numerical experiments}

\subsection{Ellipsoidal shell}

We use a portion of the ellipsoidal shell (cf. Fig. 2) and assume that the thickness of the shell is $2 \varepsilon$. In curvilinear coordinates, the middle surface is given by the mapping $\vec{\theta}$ defined by

$$
\vec{\theta}\left(y_{1}, y_{2}\right)=\left(m \sin y_{1} \cos y_{2}, n \sin y_{1} \sin y_{2}, l \cos y_{1}\right) \text {. }
$$

The domain $\omega$ is defined as follows

$$
\omega:=\left\{\left(y_{1}, y_{2}\right) \in \mathbb{R}^{2} ;\left[\frac{\pi}{6}, \frac{5 \pi}{6}\right] \times[0, \pi]\right\},
$$

and the boundary $\gamma_{0}$, where no displacement occurs, is defined

$$
\gamma_{0}:=\gamma_{01} \cup \gamma_{02} \cup \gamma_{03} \cup \gamma_{04},
$$

with

$$
\begin{aligned}
& \gamma_{01}=\left\{\left(y_{1}, y_{2}\right) \in \mathbb{R}^{2} ; y_{1} \in[0, \pi], y_{2}=\frac{5 \pi}{6}\right\}, \\
& \gamma_{02}=\left\{\left(y_{1}, y_{2}\right) \in \mathbb{R}^{2} ; y_{1}=\pi, y_{2} \in\left[\frac{\pi}{6}, \frac{5 \pi}{6}\right]\right\}, \\
& \gamma_{03}=\left\{\left(y_{1}, y_{2}\right) \in \mathbb{R}^{2} ; y_{1} \in[0, \pi], y_{2}=\frac{\pi}{6}\right\}, \\
& \gamma_{04}=\left\{\left(y_{1}, y_{2}\right) \in \mathbb{R}^{2} ; y_{1}=0, y_{2} \in\left[\frac{\pi}{6}, \frac{5 \pi}{6}\right]\right\} .
\end{aligned}
$$

Then the covariant basis of the tangent plane to $S$ at $\vec{\theta}(y)$ is given by

$$
\begin{aligned}
& \vec{a}_{1}=\frac{\partial \vec{\theta}}{\partial y_{1}}=\left(m \cos y_{1} \cos y_{2}, n \cos y_{1} \sin y_{2},-l \sin y_{1}\right), \\
& \vec{a}_{2}=\frac{\partial \vec{\theta}}{\partial y_{2}}=\left(-m \sin y_{1} \sin y_{2}, n \sin y_{1} \cos y_{2}, 0\right), \\
& \vec{a}_{3}=\left(\frac{n l \sin y_{1} \cos y_{2}}{\sqrt{Q}}, \frac{m l \sin y_{1} \sin y_{2}}{\sqrt{Q}}, \frac{m n \cos y_{1}}{\sqrt{Q}}\right),
\end{aligned}
$$



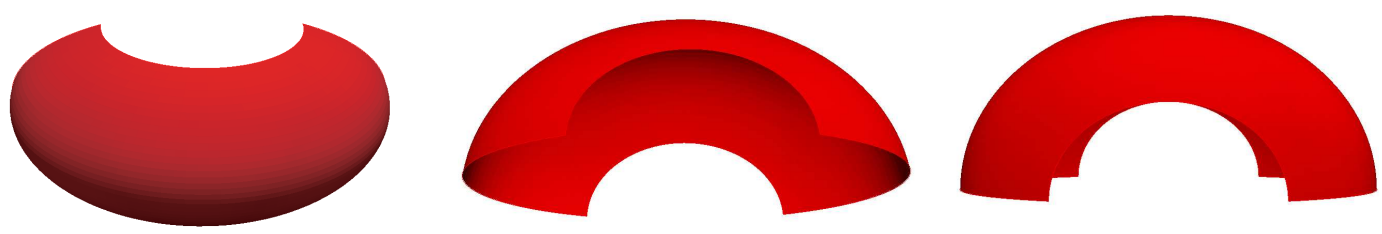

Figure 2: Portion of an ellipsoidal shell

where $Q=m^{2} n^{2} \cos ^{2} y_{1}+n^{2} l^{2} \sin ^{2} y_{1} \cos ^{2} y_{2}+m^{2} l^{2} \sin ^{2} y_{1} \sin ^{2} y_{2}$.

The covariant components of the metric tensor on $S$ are given by

$$
\begin{aligned}
& a_{11}=\vec{a}_{1} \cdot \vec{a}_{1}=m^{2} \cos ^{2} y_{1} \cos ^{2} y_{2}+n^{2} \cos ^{2} y_{1} \sin ^{2} y_{2}+l^{2} \sin ^{2} y_{1}, \\
& a_{12}=a_{21}=\vec{a}_{1} \cdot \vec{a}_{2}=\left(n^{2}-m^{2}\right) \sin y_{1} \cos y_{1} \sin y_{2} \cos y_{2}, \\
& a_{22}=\vec{a}_{2} \cdot \vec{a}_{2}=m^{2} \sin ^{2} y_{1} \sin ^{2} y_{2}+n^{2} \sin ^{2} y_{1} \cos ^{2} y_{2},
\end{aligned}
$$

and

$$
a=\operatorname{det}\left(a_{\alpha \beta}\right)=Q \sin ^{2} y_{1} .
$$

The contravariant components of the metric tensor on $S$ are given by

$$
\begin{aligned}
& a^{11}=\frac{m^{2} \sin ^{2} y_{2}+n^{2} \cos ^{2} y_{2}}{Q} \\
& a^{12}=a^{21}=\frac{\left(m^{2}-n^{2}\right) \cos y_{1} \sin y_{2} \cos y_{2}}{Q \sin y_{1}} \\
& a^{22}=\frac{m^{2} \cos ^{2} y_{1} \cos ^{2} y_{2}+n^{2} \cos ^{2} y_{1} \sin ^{2} y_{2}+l^{2} \sin ^{2} y_{1}}{Q \sin ^{2} y_{1}} .
\end{aligned}
$$

As a result,

$$
\begin{aligned}
\vec{a}^{1}= & a^{11} \vec{a}_{1}+a^{12} \vec{a}_{2} \\
= & \left(\frac{m n^{2} \cos y_{1} \cos y_{2}}{Q}, \frac{m^{2} n \cos y_{1} \sin y_{2}}{Q}, \frac{-m^{2} l \sin y_{1} \sin ^{2} y_{2}-n^{2} l \sin y_{1} \cos ^{2} y_{2}}{Q}\right), \\
\vec{a}^{2}= & a^{21} \vec{a}_{1}+a^{22} \vec{a}_{2} \\
= & \left(\frac{-m n^{2} \cos ^{2} y_{1} \sin y_{2}-m l^{2} \sin ^{2} y_{1} \sin y_{2}}{Q \sin y_{1}}, \frac{m^{2} n \cos ^{2} y_{1} \cos y_{2}+n l^{2} \sin ^{2} y_{1} \cos y_{2}}{Q \sin y_{1}},\right. \\
& \left.\frac{\left(m^{2}-n^{2}\right) l \cos ^{2} y_{1} \sin y_{2} \cos y_{2}}{Q \sin y_{1}}\right),
\end{aligned}
$$




$$
\begin{aligned}
& \partial_{1} \vec{a}_{1}=\frac{\partial \vec{a}_{1}}{\partial y_{1}}=\left(-m \sin y_{1} \cos y_{2},-n \sin y_{1} \sin y_{2},-l \cos y_{1}\right), \\
& \partial_{2} \vec{a}_{1}=\frac{\partial \vec{a}_{1}}{\partial y_{2}}=\left(-m \cos y_{1} \sin y_{2}, n \cos y_{1} \cos y_{2}, 0\right), \\
& \partial_{1} \vec{a}_{2}=\frac{\partial \vec{a}_{2}}{\partial y_{1}}=\left(-m \cos y_{1} \sin y_{2}, n \cos y_{1} \cos y_{2}, 0\right), \\
& \partial_{2} \vec{a}_{2}=\frac{\partial \vec{a}_{2}}{\partial y_{2}}=\left(-m \sin y_{1} \cos y_{2},-n \sin y_{1} \sin y_{2}, 0\right) .
\end{aligned}
$$

The covariant and mixed components of the extrinsic curvature tensor on $S$ are given by

$$
\begin{aligned}
& b_{11}=\vec{a}_{3} \cdot \partial_{1} \vec{a}_{1}=\frac{-m n l}{\sqrt{Q}}, \quad b_{12}=\vec{a}_{3} \cdot \partial_{1} \vec{a}_{2}=0, \\
& b_{21}=\vec{a}_{3} \cdot \partial_{2} \vec{a}_{1}=0, \quad b_{22}=\vec{a}_{3} \cdot \partial_{2} \vec{a}_{2}=\frac{-m n l \sin ^{2} y_{1}}{\sqrt{Q}}, \\
& b_{1}^{1}=a^{11} b_{11}+a^{12} b_{21}=\frac{-m n l\left(m^{2} \sin ^{2} y_{2}+n^{2} \cos ^{2} y_{2}\right)}{\sqrt{Q^{3}}}, \\
& b_{1}^{2}=a^{21} b_{11}+a^{22} b_{21}=0, \quad b_{2}^{1}=a^{11} b_{12}+a^{12} b_{22}=0, \\
& b_{2}^{2}=a^{21} b_{12}+a^{22} b_{22}=\frac{-m n l\left(m^{2} \cos ^{2} y_{1} \cos ^{2} y_{2}+n^{2} \cos ^{2} y_{1} \sin ^{2} y_{2}+l^{2} \sin ^{2} y_{1}\right)}{\sqrt{Q^{3}}} .
\end{aligned}
$$

The Christoffel symbols on $S$ are given by

$$
\begin{aligned}
& \Gamma_{11}^{1}=\vec{a}^{1} \cdot \partial_{1} \vec{a}_{1}=\frac{-m^{2} n^{2} \sin y_{1} \cos y_{1} \sin ^{2} y_{2}}{Q}+\frac{n^{2} l^{2} \sin y_{1} \cos y_{1} \cos ^{2} y_{2}+m^{2} l^{2} \sin y_{1} \cos y_{1} \sin ^{2} y_{2}}{Q}, \\
& \Gamma_{12}^{1}=\Gamma_{21}^{1}=0, \quad \Gamma_{22}^{1}=\vec{a}^{1} \cdot \partial_{2} \vec{a}_{2}=\frac{-m^{2} n^{2} \sin y_{1} \cos y_{1}}{Q}, \\
& \Gamma_{11}^{2}=\vec{a}^{2} \cdot \partial_{1} \vec{a}_{1}=\frac{\left(m^{2}-n^{2}\right) l^{2}\left(\sin ^{2} y_{1}-\cos ^{3} y_{1} \sin y_{2} \cos y_{2}\right)}{\sin y_{1}\left(m^{2} n^{2} \cos ^{2} y_{1}+n^{2} l^{2} \sin ^{2} y_{1} \cos ^{2} y_{2}+m^{2} l^{2} \sin ^{2} y_{1} \sin ^{2} y_{2}\right)}, \\
& \Gamma_{12}^{2}=\Gamma_{21}^{2}=\cot y_{1}, \quad \Gamma_{22}^{2}=\vec{a}^{2} \cdot \partial_{2} \vec{a}_{2}=\frac{\left(m^{2}-n^{2}\right) l^{2} \sin ^{2} y_{1} \sin y_{2} \cos y_{2}}{Q}
\end{aligned}
$$

We take the Young's modulus (cf. [2]) as $E=2.0 \times 10^{11} \mathrm{~Pa}$ and the Poisson ratio as $v=0.25$.

Since the Lamé constants, the Poisson ratio and the Young's modulus are related by (see, for instance, [14]):

$$
\lambda=\frac{E v}{(1+v)(1-2 v)}, \quad \mu=\frac{E}{2(1+v)},
$$

we get

$$
\lambda=8.0 \times 10^{10} \mathrm{~Pa}, \quad \mu=8.0 \times 10^{10} \mathrm{~Pa} .
$$




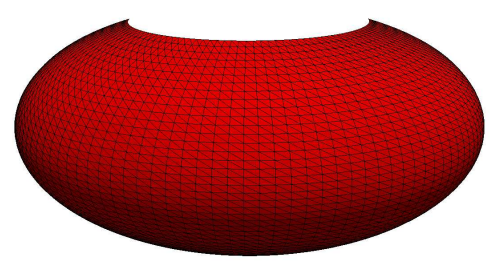

$\mathrm{n}=40, \mathrm{~m}=60$

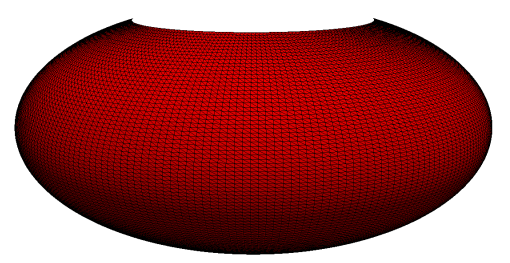

$\mathrm{n}=\mathbf{8 0}, \mathrm{m}=\mathbf{1 2 0}$

Figure 3: Meshes of the surface.

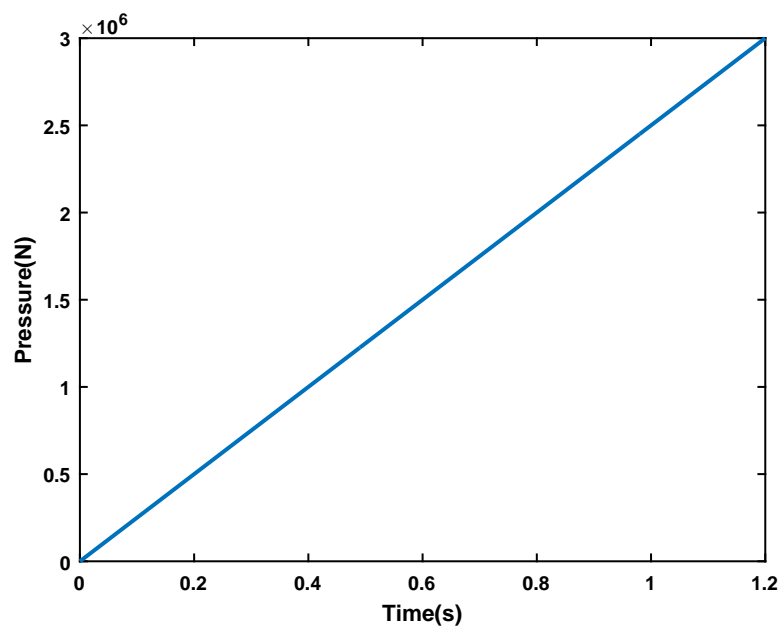

Figure 4: Applied force component $p^{2}=2.5 \times 10^{6} t, t \in[0,1.2]$.

We assume the shell to be made of stainless steel, whose mass density is $\rho=7.8 \times 10^{3} \mathrm{~kg} / \mathrm{m}^{3}$.

We are now in a position to solve the time-dependent problem by using the Newmark scheme with the software FreeFEM++ (cf. [28]) and we visualize the output using ParaView (cf. [32]). Two meshes comprising of 2,400 and 9,600 elements used for the computation are shown in Fig. 3.

The set of parameters related to the numerical experiments is the following: $m=$ $0.06 \mathrm{~m}, n=0.05 \mathrm{~m}, l=0.03 \mathrm{~m}$, semi-thickness $\varepsilon=0.001 \mathrm{~m}$ (cf. [2]), Young's modulus $E=2.0 \times$ $10^{11} \mathrm{~Pa}$, Poisson ratio $v=0.25$, mass density $\rho=7.8 \times 10^{3} \mathrm{~kg} / \mathrm{m}^{3}$ and $\gamma=0.6, \beta=(1 / 2+\gamma)^{2} / 4$. Let the external force be $p^{1}=p^{3}=0$ whereas $p^{2}=f(t)=2.5 \times 10^{6} t, t \in[0,1.2]$ (see Fig. 4).

The deformations of the shell at different times are shown in Figs. 5 and 6 (from different angles), for the four combinations of meshes $(n, m)=(40,60)$ and $(n, m)=(80,120)$, time-steps $\Delta t=0.01 \mathrm{~s}$ and $\Delta t=0.005 \mathrm{~s}$. To show that the entire boundary is clamped, we provide the figures from other angle (see Fig. 6). The entire lateral of each figure is in blue color, indicating that the entire boundary is clamped, whereas the biggest deformation happens on the parts in red color, i.e., the center of the ellipsoidal shell. 

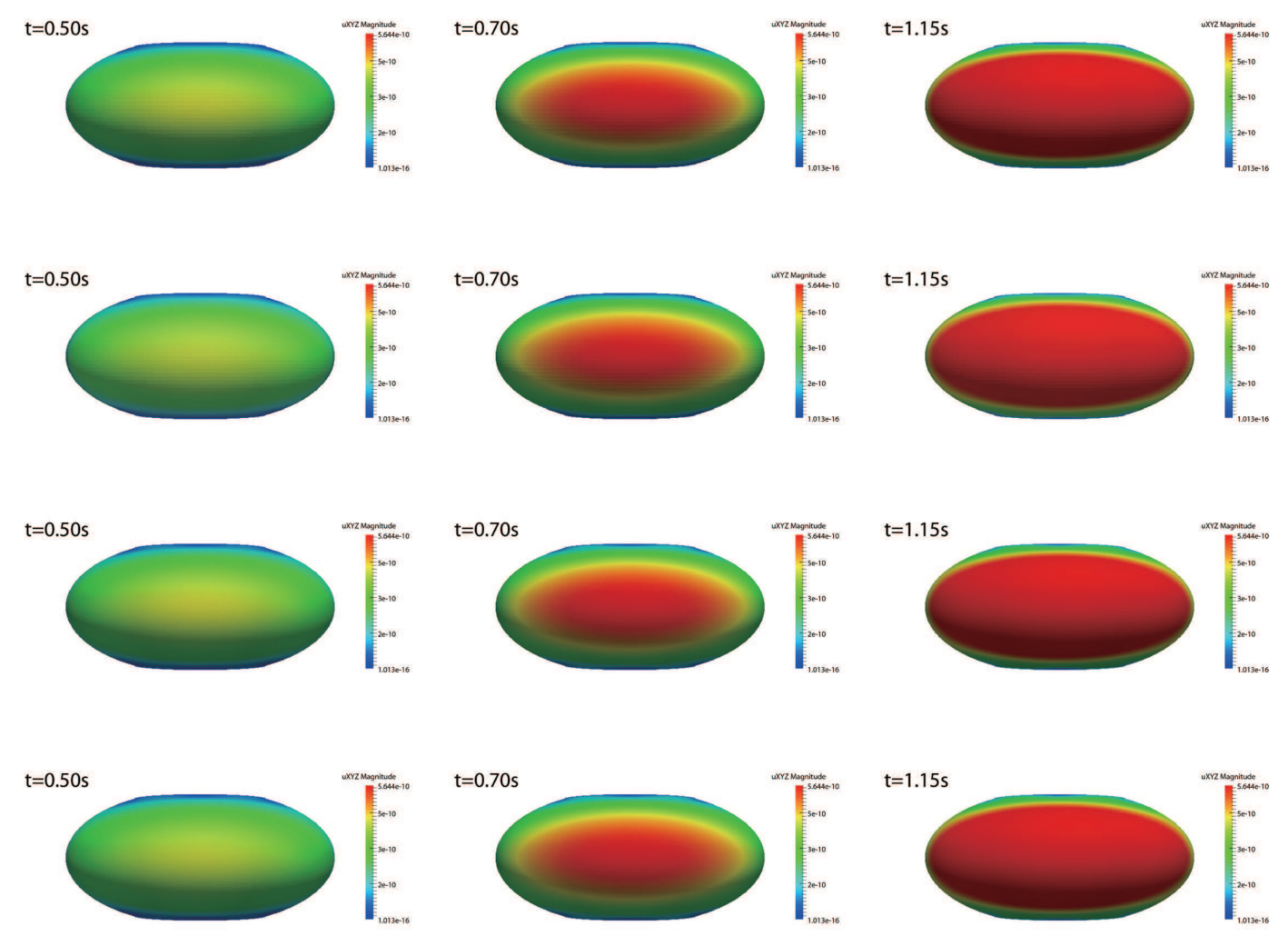

Figure 5: Deformations of the ellipsoidal shell at different times. Different mesh $(n, m)=(40,60)$ (in the first and second line) and $(\mathrm{n}, \mathrm{m})=(80,120)$ (in the third and fourth line), different time-step $\Delta t=0.01 \mathrm{~s}$ (in the first and third line) and $\Delta t=0.005 \mathrm{~s}$ (in the second and fourth line) respectively.

To illustrate the deformation of the shell we take three random points for each mesh. Tables 1-3 show the displacements of three random points at time $t=0.50 \mathrm{~s}, t=0.70 \mathrm{~s}$ and $t=1.15 \mathrm{~s}$, respectively.

The consistent shapes in Figs. 5 and 6 indicate that the numerical scheme for timedependent elliptic membrane shell model with ellipsoidal middle surface is stable under the different combinations of time-step and space-step. The consistent displacements in Tables 1-3 indicate the same stability under different time-steps. This is in accordance with the conclusion of our Theorem 5.1, which infers the stability of the adopted numerical scheme. Moreover, the deformations of the middle surface match the change in applied force, which corroborates the efficiency of the model.

Moreover, we concern the convergent orders of $\vec{L}^{2}$ norm related to the theoretical result of Theorem 5.1. Based on the fact the exact solution of the model cannot be derived, we must replace the exact solution with the approximate solution in refined space and 

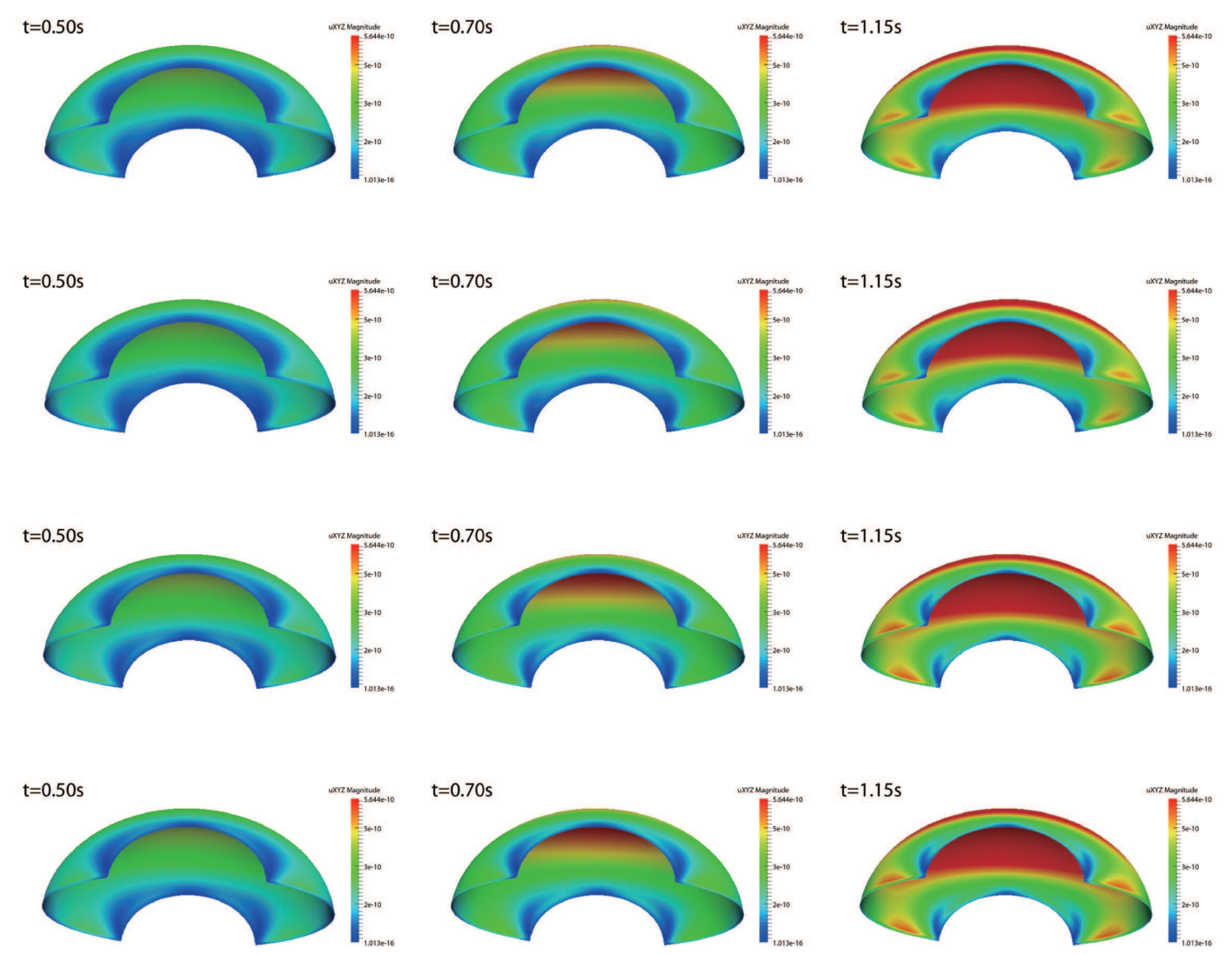

Figure 6: Deformations of the ellipsoidal shell at different times from other angle. Different mesh $(\mathrm{n}, \mathrm{m})=(40,60)$ (in the first and second line) and $(\mathrm{n}, \mathrm{m})=(80,120)$ (in the third and fourth line), different time-step $\Delta t=0.01 \mathrm{~s}$ (in the first and third line) and $\Delta t=0.005 \mathrm{~s}$ (in the second and fourth line) respectively.

Table 1: Displacements of three random points at time $t=0.50 \mathrm{~s}$.

\begin{tabular}{|c|c|c|c|c|c|c|c|}
\hline \multirow{2}{*}{\multicolumn{2}{|c|}{$\begin{array}{c}\text { space-step } \\
\text { time-step }\end{array}$}} & \multicolumn{2}{|c|}{ meshes $40 \times 60$} & \multirow{2}{*}{\multicolumn{2}{|c|}{$\begin{array}{c}\text { space-step } \\
\text { time-step }\end{array}$}} & \multicolumn{2}{|c|}{ meshes $80 \times 120$} \\
\hline & & $\Delta t=0.01 \mathrm{~s}$ & $\Delta t=0.005 \mathrm{~s}$ & & & $\Delta t=0.01 \mathrm{~s}$ & $\Delta t=0.005 \mathrm{~s}$ \\
\hline \multirow{3}{*}{ point1 } & $x$ & $-1.13053761 \mathrm{e}-010$ & $-1.10879653 e-010$ & \multirow{3}{*}{ point1 } & $x$ & $-1.00017938 \mathrm{e}-010$ & $-9.80945186 \mathrm{e}-011$ \\
\hline & $\mathrm{y}$ & $1.01491309 \mathrm{e}-012$ & $9.95395498 \mathrm{e}-013$ & & $\mathrm{y}$ & $1.15143084 \mathrm{e}-012$ & $1.12928796 \mathrm{e}-012$ \\
\hline & $\mathrm{z}$ & $-2.28152913 e-010$ & $-2.23765367 \mathrm{e}-010$ & & $\mathrm{z}$ & $-2.01242856 \mathrm{e}-010$ & $-1.97372799 \mathrm{e}-010$ \\
\hline \multirow{3}{*}{ point2 } & $x$ & $-1.21817084 \mathrm{e}-010$ & $-1.19474458 \mathrm{e}-010$ & \multirow{3}{*}{ point2 } & $x$ & $-1.10726650 \mathrm{e}-010$ & $-1.08597291 \mathrm{e}-010$ \\
\hline & $\mathrm{y}$ & $1.38090245 \mathrm{e}-012$ & $1.35434665 \mathrm{e}-012$ & & $\mathrm{y}$ & $1.48735722 \mathrm{e}-012$ & $1.45875423 \mathrm{e}-012$ \\
\hline & $\mathrm{z}$ & $-2.21076837 \mathrm{e}-010$ & $-2.16825363 e-010$ & & $\mathrm{z}$ & $-2.00407260 \mathrm{e}-010$ & $-1.96553274 \mathrm{e}-010$ \\
\hline \multirow{3}{*}{ point3 } & $x$ & $-1.27404143 e-010$ & $-1.24954061 \mathrm{e}-010$ & \multirow{3}{*}{ point3 } & $\mathrm{x}$ & $-1.26771219 \mathrm{e}-010$ & $-1.24333308 \mathrm{e}-010$ \\
\hline & $\mathrm{y}$ & $1.80589061 \mathrm{e}-012$ & $1.77116188 \mathrm{e}-012$ & & $\mathrm{y}$ & $1.75935731 \mathrm{e}-012$ & $1.72552359 \mathrm{e}-012$ \\
\hline & $\mathrm{z}$ & $-2.07770232 \mathrm{e}-010$ & $-2.03774650 \mathrm{e}-010$ & & $\mathrm{z}$ & $-1.46849435 e-010$ & $-1.44025417 \mathrm{e}-010$ \\
\hline
\end{tabular}


Table 2: Displacements of three random points at time $t=0.70 \mathrm{~s}$.

\begin{tabular}{|c|c|c|c|c|c|c|c|}
\hline \multirow{2}{*}{\multicolumn{2}{|c|}{$\begin{array}{l}\text { space-step } \\
\text { time-step }\end{array}$}} & \multicolumn{2}{|c|}{ meshes $40 \times 60$} & \multirow{2}{*}{\multicolumn{2}{|c|}{\begin{tabular}{|l|} 
space-step \\
time-step
\end{tabular}}} & \multicolumn{2}{|c|}{ meshes $80 \times 120$} \\
\hline & & $\Delta t=0.01 \mathrm{~s}$ & $\Delta t=0.005 \mathrm{~s}$ & & & $\Delta t=0.01 \mathrm{~s}$ & $\Delta t=0.005 \mathrm{~s}$ \\
\hline \multirow{3}{*}{ point1 } & $x$ & $-1.56535979 \mathrm{e}-010$ & $-1.54361871 \mathrm{e}-010$ & \multirow{3}{*}{ point1 } & $x$ & $-1.07175047 \mathrm{e}-010$ & $-1.05686501 \mathrm{e}-010$ \\
\hline & $\mathrm{y}$ & $1.40526425 \mathrm{e}-012$ & $1.38574666 \mathrm{e}-012$ & & $\mathrm{y}$ & $1.04077782 \mathrm{e}-012$ & $1.02632258 \mathrm{e}-012$ \\
\hline & $\mathrm{z}$ & $-3.15904053 e-010$ & $-3.11516479 e-010$ & & $\mathrm{z}$ & $-2.52999260 \mathrm{e}-010$ & $-2.49485405 \mathrm{e}-010$ \\
\hline \multirow{3}{*}{ point2 } & $x$ & $-1.68669814 \mathrm{e}-010$ & $-1.66327174 \mathrm{e}-010$ & \multirow{3}{*}{ point2 } & $x$ & $-1.29332711 \mathrm{e}-010$ & $-1.27536426 \mathrm{e}-010$ \\
\hline & $\mathrm{y}$ & $1.91201872 \mathrm{e}-012$ & $1.88546292 \mathrm{e}-012$ & & $\mathrm{y}$ & $1.39090052 \mathrm{e}-012$ & $1.37158254 \mathrm{e}-012$ \\
\hline & $\mathrm{z}$ & $-3.06106390 \mathrm{e}-010$ & $-3.01854930 \mathrm{e}-010$ & & $\mathrm{z}$ & $-2.74387763 e-010$ & $-2.70576839 \mathrm{e}-010$ \\
\hline \multirow{3}{*}{ point3 } & $x$ & $-1.76405723 e-010$ & $-1.73955655 e-010$ & \multirow{3}{*}{ point3 } & $x$ & $-1.38486375 e-010$ & $-1.36562955 \mathrm{e}-010$ \\
\hline & $\mathrm{y}$ & $2.50046381 \mathrm{e}-012$ & $2.46573530 \mathrm{e}-012$ & & $\mathrm{y}$ & $1.59428883 \mathrm{e}-012$ & $1.57214595 \mathrm{e}-012$ \\
\hline & $\mathrm{z}$ & $-2.87681851 e-010$ & $-2.83686269 \mathrm{e}-010$ & & $\mathrm{z}$ & $-2.78643941 e-010$ & $-2.74773898 \mathrm{e}-010$ \\
\hline
\end{tabular}

Table 3: Displacements of three random points at time $t=1.15 \mathrm{~s}$.

\begin{tabular}{|c|c|c|c|c|c|c|c|}
\hline \multirow{2}{*}{\multicolumn{2}{|c|}{$\begin{array}{c}\text { space-step } \\
\text { time-step }\end{array}$}} & \multicolumn{2}{|c|}{ meshes $40 \times 60$} & \multirow{2}{*}{\multicolumn{2}{|c|}{$\begin{array}{c}\text { space-step } \\
\text { time-step }\end{array}$}} & \multicolumn{2}{|c|}{ meshes $80 \times 120$} \\
\hline & & $\Delta t=0.01 \mathrm{~s}$ & $\Delta t=0.005 \mathrm{~s}$ & & & $\Delta t=0.01 \mathrm{~s}$ & $\Delta t=0.005 \mathrm{~s}$ \\
\hline \multirow{3}{*}{ point1 } & $x$ & $-2.68803702 \mathrm{e}-010$ & $-2.66506234 \mathrm{e}-010$ & \multirow{3}{*}{ point1 } & $x$ & $-2.72280282 \mathrm{e}-010$ & $-2.69953115 e-010$ \\
\hline & $\mathrm{y}$ & $1.01072891 \mathrm{e}-011$ & $1.00209025 \mathrm{e}-011$ & & $\mathrm{y}$ & $1.05072444 \mathrm{e}-011$ & $1.04174386 \mathrm{e}-011$ \\
\hline & $\mathrm{z}$ & $-3.93507393 e-010$ & $-3.90144084 \mathrm{e}-010$ & & $\mathrm{z}$ & $-2.44796405 e-010$ & $-2.42704135 e-010$ \\
\hline \multirow{3}{*}{ point2 } & $x$ & $-2.82350060 \mathrm{e}-010$ & $-2.79936796 \mathrm{e}-010$ & \multirow{3}{*}{ point2 } & $x$ & $-2.67804612 \mathrm{e}-010$ & $-2.65515693 e-010$ \\
\hline & $\mathrm{y}$ & $1.70157898 \mathrm{e}-011$ & $1.68703558 \mathrm{e}-011$ & & $\mathrm{y}$ & $1.12692147 \mathrm{e}-011$ & $1.11728968 \mathrm{e}-011$ \\
\hline & $\mathrm{z}$ & $-2.91695307 \mathrm{e}-010$ & $-2.89202190 \mathrm{e}-010$ & & $\mathrm{z}$ & $-2.24397015 e-010$ & $-2.22479091 e-010$ \\
\hline \multirow{3}{*}{ point3 } & $x$ & $-2.57885935 e-010$ & $-2.55681781 \mathrm{e}-010$ & \multirow{3}{*}{ point3 } & $x$ & $-2.62833533 e-010$ & $-2.60587080 \mathrm{e}-010$ \\
\hline & $\mathrm{y}$ & $1.22005366 \mathrm{e}-011$ & $1.20962589 \mathrm{e}-011$ & & $\mathrm{y}$ & $1.20429743 \mathrm{e}-011$ & $1.19400436 \mathrm{e}-011$ \\
\hline & $\mathrm{z}$ & $-2.00858913 e-010$ & $-1.99142175 \mathrm{e}-010$ & & $\mathrm{z}$ & $-2.04538914 \mathrm{e}-010$ & $-2.02790715 e-010$ \\
\hline
\end{tabular}

Table 4: Convergent order and error with different mesh size $(\gamma=0.6, \Delta t=0.0001 \mathrm{~s})$.

\begin{tabular}{||ccc||}
\hline mesh & $\left\|\vec{\zeta}_{h}^{n}-\vec{\zeta}\left(t_{n}\right)\right\|_{\vec{L}^{2}}$ & order \\
\hline $10 \times 15$ & $5.96754 \mathrm{e}-013$ & $/$ \\
$20 \times 30$ & $3.09816 \mathrm{e}-013$ & 0.96 \\
$40 \times 60$ & $1.56949 \mathrm{e}-013$ & 0.98 \\
$80 \times 120$ & $7.98694 \mathrm{e}-014$ & 0.97 \\
$160 \times 240$ & $4.2221 \mathrm{e}-014$ & 0.92 \\
\hline
\end{tabular}

time steps (meshes: $530 \times 795$, time-steps: $\Delta t=0.0001 \mathrm{~s}$ ) to verify the convergent orders. Under the stable condition of $\gamma>1 / 2$, we take $\gamma=0.6$. We can find that convergent rates with different mesh size and time-steps are nearly first order which can be found in Table 4, Table 5, Fig. 7 and Fig. 8. 
Table 5: Convergent order and error with different time-step $(\gamma=0.6$, mesh: $530 \times 795)$.

\begin{tabular}{||ccc||}
\hline$\Delta \mathrm{t}$ & $\left\|\vec{\zeta}_{h}^{n}-\vec{\zeta}\left(t_{n}\right)\right\|_{\vec{L}^{2}}$ & order \\
\hline $0.005 \mathrm{~s}$ & $1.94235 \mathrm{e}-012$ & $/$ \\
$0.0025 \mathrm{~s}$ & $9.51355 \mathrm{e}-013$ & 1.03 \\
$0.00125 \mathrm{~s}$ & $4.55857 \mathrm{e}-013$ & 1.06 \\
$0.000625 \mathrm{~s}$ & $2.08109 \mathrm{e}-013$ & 1.13 \\
$0.0003125 \mathrm{~s}$ & $8.42346 \mathrm{e}-014$ & 1.30 \\
\hline
\end{tabular}

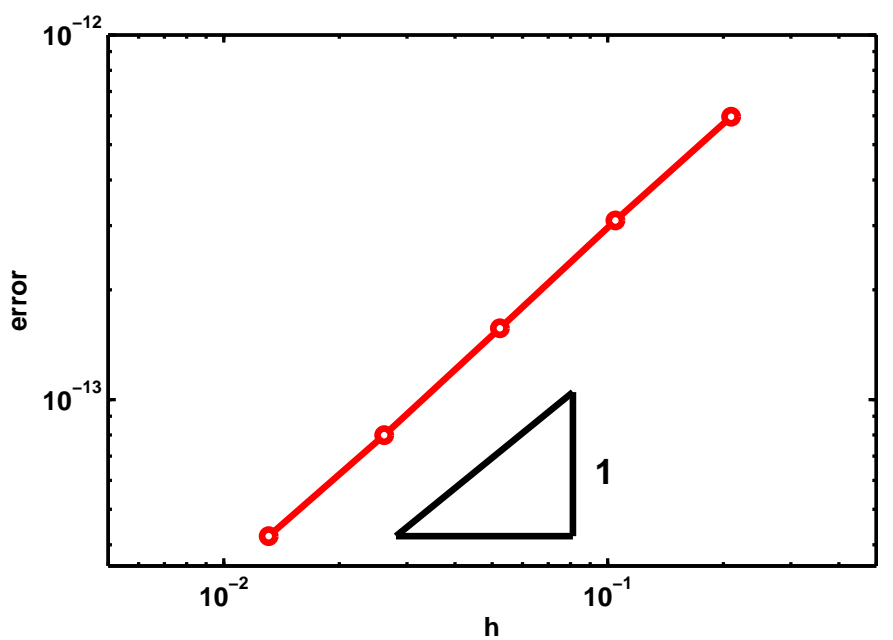

Figure 7: Convergence of errors with respect to mesh size $(\gamma=0.6, \Delta t=0.0001 \mathrm{~s})$.

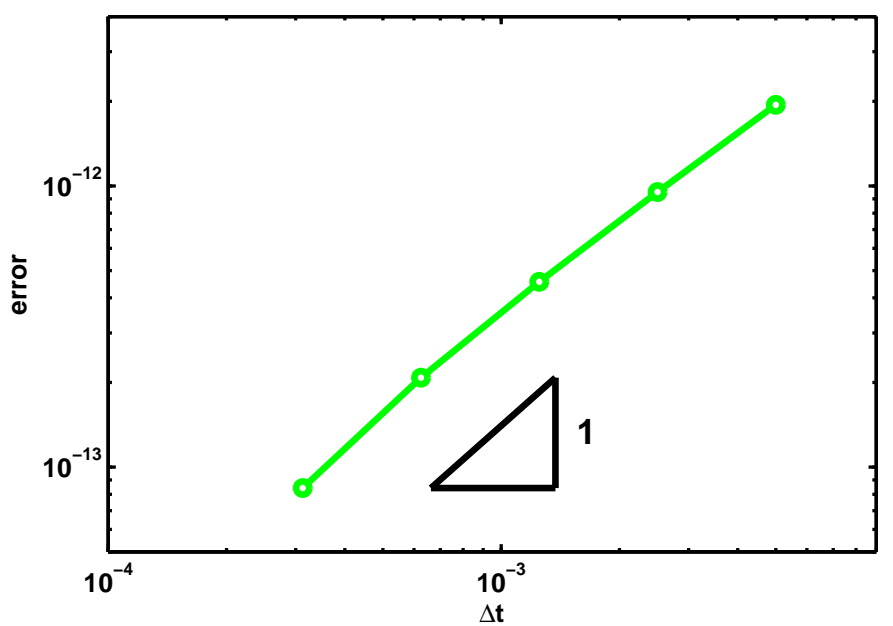

Figure 8: Convergence of errors with respect to time step $(\gamma=0.6$, mesh:530 $\times 795)$. 


\subsection{Spherical shell}

We use a portion of the spherical shell (cf. Fig. 9) and assume that the thickness of the shell is $2 \varepsilon$. In curvilinear coordinates, the middle surface is given by the mapping $\vec{\theta}$ defined by

$$
\vec{\theta}\left(y_{1}, y_{2}\right)=\left(r \cos y_{1} \sin y_{2}, r \sin y_{1} \sin y_{2}, r \cos y_{2}\right) .
$$

Here

$$
\omega:=\left\{\left(y_{1}, y_{2}\right) \in \mathbb{R}^{2} ; 0 \leq y_{1} \leq \pi, \epsilon \leq y_{2} \leq \pi / 2\right\},
$$

where $\epsilon$ is very small positive constant, and

$$
\gamma_{0}:=\gamma_{01} \cup \gamma_{02} \cup \gamma_{03} \cup \gamma_{04},
$$

where

$$
\begin{aligned}
& \gamma_{01}=\left\{\left(y_{1}, y_{2}\right) \in \mathbb{R}^{2} ; 0 \leq y_{1} \leq \pi, y_{2}=\epsilon\right\}, \\
& \gamma_{02}=\left\{\left(y_{1}, y_{2}\right) \in \mathbb{R}^{2} ; y_{1}=\pi, \epsilon \leq y_{2} \leq \pi / 2\right\}, \\
& \gamma_{03}=\left\{\left(y_{1}, y_{2}\right) \in \mathbb{R}^{2} ; 0 \leq y_{1} \leq \pi, y_{2}=\pi / 2\right\}, \\
& \gamma_{04}=\left\{\left(y_{1}, y_{2}\right) \in \mathbb{R}^{2} ; y_{1}=0, \epsilon \leq y_{1} \leq \pi / 2\right\}
\end{aligned}
$$

Then the covariant basis of the tangent plane to $S$ at $\vec{\theta}(y)$ is given by

$$
\begin{aligned}
& \vec{a}_{1}=\frac{\partial \vec{\theta}}{\partial y_{1}}=\left(-r \sin y_{1} \sin y_{2}, r \sin y_{1} \cos y_{2}, 0\right), \\
& \vec{a}_{2}=\frac{\partial \vec{\theta}}{\partial y_{2}}=\left(r \cos y_{1} \cos y_{2}, r \cos y_{1} \sin y_{2},-r \sin y_{1}\right), \\
& \vec{a}_{3}=\vec{a}^{3}=\left(\cos y_{1} \sin y_{2}, \sin y_{1} \sin y_{2}, \cos y_{2}\right) .
\end{aligned}
$$

The covariant components of metric tensors on $S$ are given by

$$
a_{11}=\vec{a}_{1} \cdot \vec{a}_{1}=r^{2} \sin ^{2} y_{2}, \quad a_{21}=a_{12}=0, \quad a_{22}=\vec{a}_{2} \cdot \vec{a}_{2}=r^{2},
$$
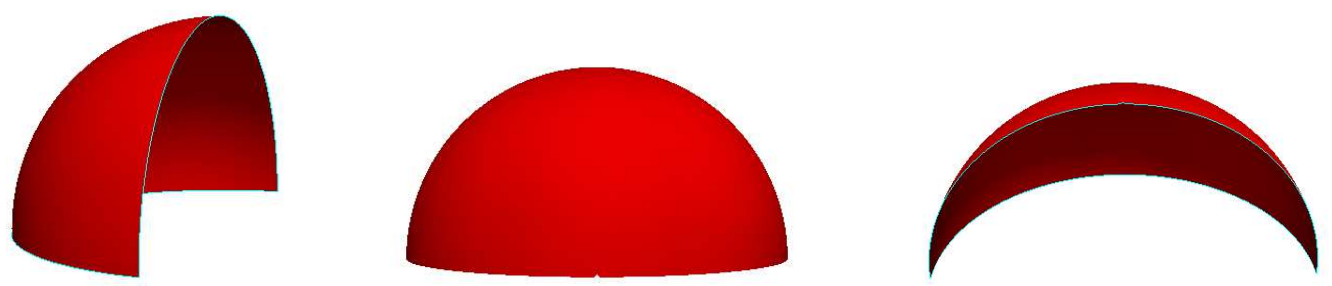

Figure 9: Portion of a spherical shell. 
and

$$
a=\operatorname{det}\left(a_{\alpha \beta}\right)=r^{4} \sin ^{2} y_{2} .
$$

The contravariant components of metric tensors on $S$ are given by

$$
a^{11}=\left(a_{11}\right)^{-1}=r^{-2} \sin ^{-2} y_{2}, \quad a^{21}=a^{12}=0, \quad a^{22}=\left(a_{22}\right)^{-1}=r^{-2} .
$$

It can be immediately noticed that the contravariant component $a^{11}$ is not defined at $y_{2}=0$. As a result,

$$
\begin{aligned}
& \vec{a}^{1}=a^{1 \alpha} \cdot \vec{a}_{\alpha}=\left(-r^{-1} \sin y_{1} \sin ^{-1} y_{2}, r^{-1} \cos y_{1} \sin ^{-1} y_{2}, 0\right), \\
& \vec{a}^{2}=a^{2 \alpha} \cdot \vec{a}_{\alpha}=\left(r^{-1} \cos y_{1} \cos y_{2}, r^{-1} \sin y_{1} \cos y_{2},-r^{-1} \sin y_{2}\right), \\
& \partial_{1} \overrightarrow{a_{1}}=\frac{\partial \overrightarrow{a_{1}}}{\partial y_{1}}=\left(-r \cos y_{1} \sin y_{2},-r \sin y_{1} \sin y_{2}, 0\right), \\
& \partial_{2} \overrightarrow{a_{1}}=\partial_{1} \overrightarrow{a_{2}}=\frac{\partial \overrightarrow{a_{1}}}{\partial y_{2}}=\left(-r \sin y_{1} \cos y_{2}, r \cos y_{1} \cos y_{2}, 0\right), \\
& \partial_{2} \overrightarrow{a_{2}}=\frac{\partial \overrightarrow{a_{2}}}{\partial y_{2}}=\left(-r \cos y_{1} \sin y_{2},-r \sin y_{1} \sin y_{2},-r \cos y_{2}\right) .
\end{aligned}
$$

The covariant and mixed components of extrinsic curvature tensors on $S$ are given by

$$
\begin{aligned}
& b_{11}=\vec{a}_{3} \cdot \partial_{1} \overrightarrow{a_{1}}=-r \sin ^{2} y_{2}, \quad b_{12}=b_{21}=0, \quad b_{22}=\vec{a}_{3} \cdot \partial_{2} \overrightarrow{a_{2}}=-r, \\
& b_{1}^{1}=a^{1 \alpha} b_{1 \alpha}=-r^{-1}, \quad b_{1}^{2}=b_{2}^{1}=0, \quad b_{2}^{2}=a^{2 \alpha} b_{2 \alpha}=-r^{-1} .
\end{aligned}
$$

The Christoffel symbols on $S$ are given by

$$
\begin{array}{ll}
\Gamma_{21}^{1}=\sin ^{-1} y_{2} \cos y_{2}, & \Gamma_{12}^{1}=\sin ^{-1} y_{2} \cos y_{2}, \\
\Gamma_{11}^{2}=-\sin y_{2} \cos y_{2}, & \Gamma_{11}^{1}=\Gamma_{22}^{1}=\Gamma_{12}^{2}=\Gamma_{21}^{2}=\Gamma_{22}^{2}=0 .
\end{array}
$$

According to [31], we choose the Young's modulus as $E=1.15 \times 10^{11} \mathrm{~Pa}$ and the Poisson ratio as $\nu=0.3$, we get $\lambda=6.6346 \times 10^{10} \mathrm{~Pa}, \mu=4.4231 \times 10^{10} \mathrm{~Pa}$. We assume the shell to be made of cast iron, whose mass density is, approximately $\rho=6.95 \mathrm{~kg} / \mathrm{m}^{3}$. Now we solve the time-dependent problem with FreeFEM++ (cf. [28]) and we visualize the results using ParaView (cf. [32]). Two meshes consisting of 5,000 and 20,000 elements used for the computation are shown in Fig. 10. We set $r=6 \mathrm{~m}$, semi-thickness $\varepsilon=0.000025 \mathrm{~m}$ (cf. [31]), $\gamma=0.6, \beta=(1 / 2+\gamma)^{2} / 4$. Let the external force $p^{1}=p^{3}=0$ whereas $p^{2}=f(t)=400 \sin t$, $t \in[0, \pi / 2]$ is shown in Fig. 11.

The deformations of the shell at different times are shown in Fig. 12, respectively, for the time-step $\Delta t=0.01 \mathrm{~s}$ and $\Delta t=0.005 \mathrm{~s}$, for the space-step $(\mathrm{n}, \mathrm{m})=(100,50)$ and $(\mathrm{n}, \mathrm{m})=$ $(200,100)$. The blue color indicates the clamping, whereas the biggest deformation takes place on the parts in red color.

To illustrate the deformation of the shell numerically, we take three random points for each mesh. Tables 6-8 show the displacements of the three random points at time $t=0.25 \mathrm{~s}, t=0.30 \mathrm{~s}$ and $t=0.43 \mathrm{~s}$, respectively. 


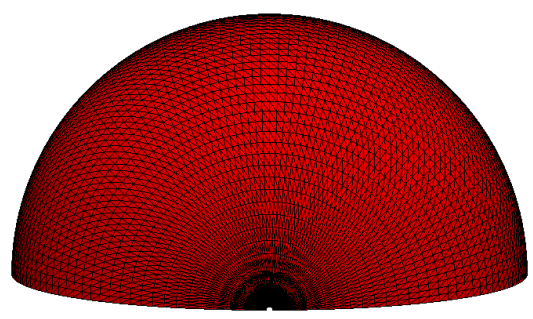

$m=100, n=50$

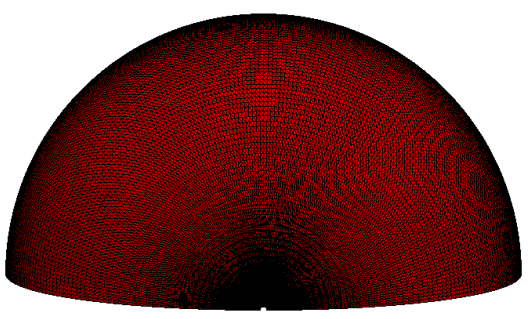

$\mathrm{m}=200, \mathrm{n}=100$

Figure 10: Meshes of the surface.

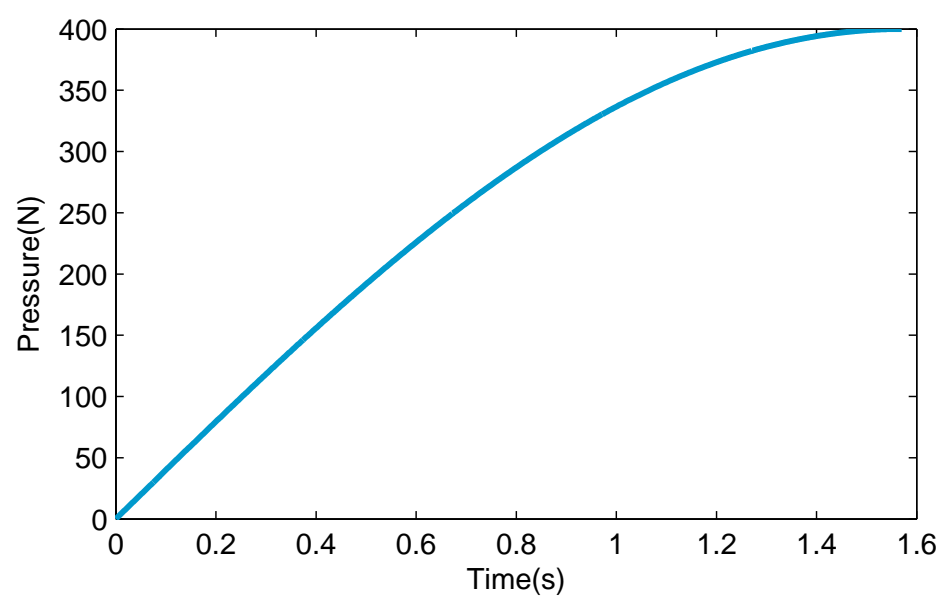

Figure 11: Applied force component $p^{2}=400 \sin t, t \in[0, \pi / 2]$.

Table 6: Displacements of three random points at time $t=0.25 \mathrm{~s}$.

\begin{tabular}{|c|c|c|c|c|c|c|c|}
\hline \multirow{2}{*}{\multicolumn{2}{|c|}{$\frac{\text { space-step }}{\text { time-step }}$}} & \multicolumn{2}{|c|}{ meshes $100 \times 50$} & \multirow{2}{*}{\multicolumn{2}{|c|}{$\begin{array}{c}\text { space-step } \\
\text { time-step }\end{array}$}} & \multicolumn{2}{|c|}{ meshes $200 \times 100$} \\
\hline & & $\Delta t=0.01 \mathrm{~s}$ & $\Delta t=0.005 \mathrm{~s}$ & & & $\Delta t=0.01 \mathrm{~s}$ & $\Delta t=0.005 \mathrm{~s}$ \\
\hline \multirow{3}{*}{ point1 } & $x$ & $-1.24158886 \mathrm{e}-011$ & $-1.19666646 \mathrm{e}-011$ & \multirow{3}{*}{ point1 } & $x$ & $-1.72509004 \mathrm{e}-011$ & $-1.66267364 \mathrm{e}-011$ \\
\hline & $\mathrm{y}$ & $4.08067365 \mathrm{e}-011$ & $3.93302751 \mathrm{e}-011$ & & $\mathrm{y}$ & $4.85795605 \mathrm{e}-011$ & $4.68218658 \mathrm{e}-011$ \\
\hline & $\mathrm{z}$ & $-1.74695458 \mathrm{e}-009$ & $-1.68374692 \mathrm{e}-009$ & & $\mathrm{z}$ & $-2.04065231 \mathrm{e}-009$ & $-1.96681804 \mathrm{e}-009$ \\
\hline \multirow{3}{*}{ point2 } & $x$ & $-4.69416901 \mathrm{e}-011$ & $-4.52432709 \mathrm{e}-011$ & \multirow{3}{*}{ point2 } & $x$ & $-5.14694294 \mathrm{e}-011$ & $-4.96071829 \mathrm{e}-011$ \\
\hline & $\mathrm{y}$ & $1.16826618 \mathrm{e}-010$ & $1.12599610 \mathrm{e}-010$ & & $\mathrm{y}$ & $1.07997507 \mathrm{e}-010$ & $1.04089959 \mathrm{e}-010$ \\
\hline & $\mathrm{z}$ & $-5.59017188 \mathrm{e}-009$ & $-5.38791012 e-009$ & & $\mathrm{z}$ & $-5.35582156 e-009$ & $-5.16203880 e-009$ \\
\hline \multirow{3}{*}{ point3 } & $x$ & $-9.31898655 e-011$ & $-8.98181113 \mathrm{e}-011$ & \multirow{3}{*}{ point3 } & $\bar{x}$ & $-9.73588501 e-011$ & $-9.38362513 e-011$ \\
\hline & $\mathrm{y}$ & $1.83493248 \mathrm{e}-010$ & $1.76854129 \mathrm{e}-010$ & & $\mathrm{y}$ & $1.59115138 \mathrm{e}-010$ & $1.53358062 \mathrm{e}-010$ \\
\hline & $\mathrm{z}$ & $-9.87763382 \mathrm{e}-009$ & $-9.52024415 e-009$ & & $\mathrm{z}$ & $-9.32391941 e-009$ & $-8.98656527 \mathrm{e}-009$ \\
\hline
\end{tabular}



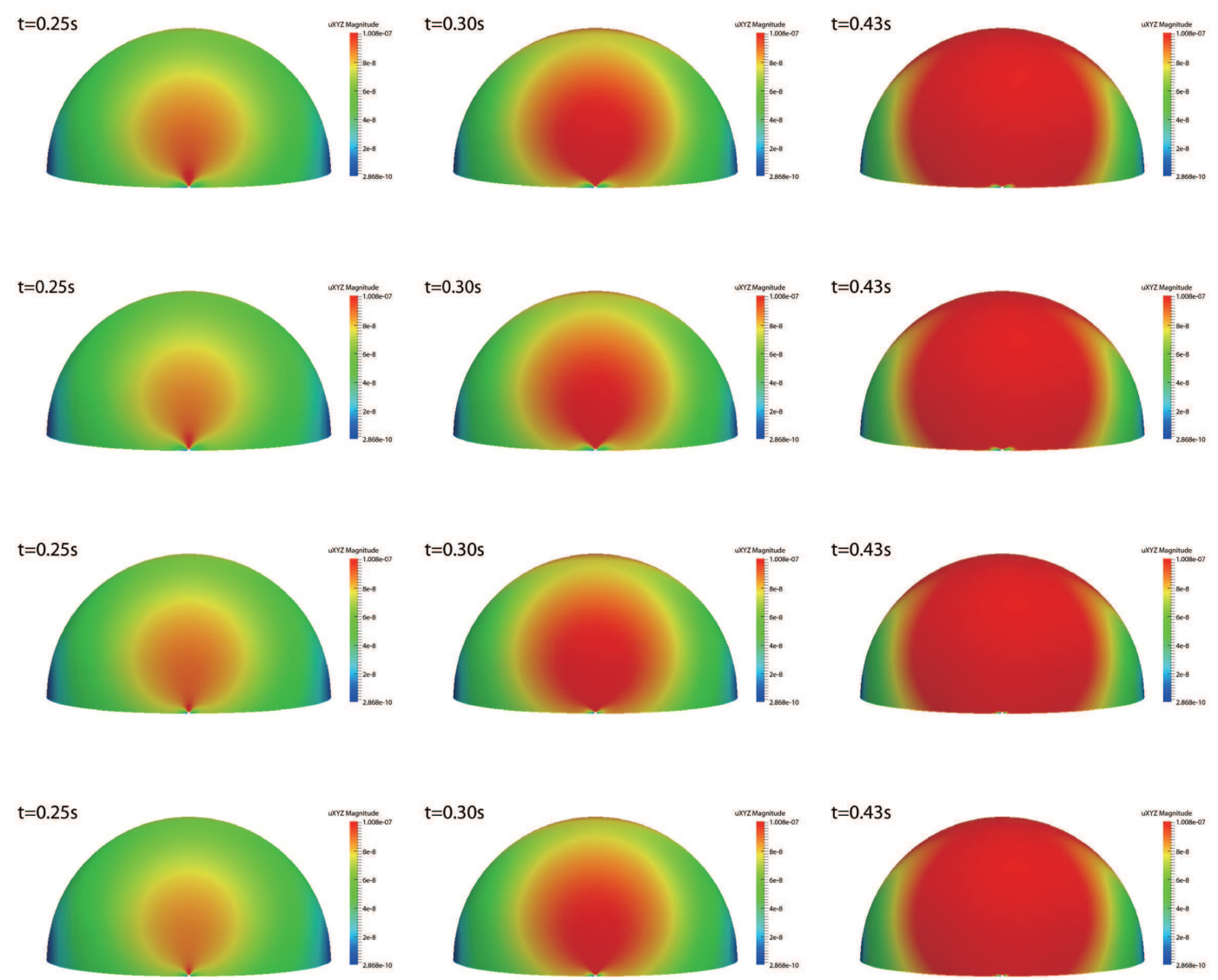

Figure 12: Deformations of the spherical shell at different times. $(n, m)=(100,50)$ (in the first and second line) and $(\mathrm{n}, \mathrm{m})=(200,100)$ (in the third and fourth line), $\Delta t=0.01 \mathrm{~s}$ (in the first and third line) and $\Delta t=0.005 \mathrm{~s}$ (in the second and fourth line) respectively.

Table 7: Displacements of three random points at time $t=0.30 \mathrm{~s}$.

\begin{tabular}{|c|c|c|c|c|c|c|c|}
\hline \multirow{2}{*}{\multicolumn{2}{|c|}{$\frac{\text { space-step }}{\text { time-step }}$}} & \multicolumn{2}{|c|}{ meshes $100 \times 50$} & \multirow{2}{*}{\multicolumn{2}{|c|}{$\begin{array}{l}\text { space-step } \\
\text { time-step }\end{array}$}} & \multicolumn{2}{|c|}{ meshes $200 \times 100$} \\
\hline & & $\Delta t=0.01 \mathrm{~s}$ & $\Delta t=0.005 \mathrm{~s}$ & & & $\Delta t=0.01 \mathrm{~s}$ & $\Delta t=0.005 \mathrm{~s}$ \\
\hline \multirow{3}{*}{ point1 } & $x$ & $-3.31608491 e-011$ & $-3.21585397 e-011$ & \multirow{3}{*}{ point1 } & $\mathrm{x}$ & $-3.86991134 \mathrm{e}-011$ & $-3.75294067 e-011$ \\
\hline & $\mathrm{y}$ & $9.41673128 \mathrm{e}-011$ & $9.13210549 \mathrm{e}-011$ & & $\mathrm{y}$ & $9.33820729 \mathrm{e}-011$ & $9.05595460 \mathrm{e}-011$ \\
\hline & $\mathrm{z}$ & $-4.25892832 \mathrm{e}-009$ & $-4.13019974 \mathrm{e}-009$ & & $\mathrm{z}$ & $-4.26283808 \mathrm{e}-009$ & $-4.13399137 \mathrm{e}-009$ \\
\hline \multirow{3}{*}{ point2 } & $\mathrm{x}$ & $-8.10228620 \mathrm{e}-011$ & -7.85738904 & \multirow{3}{*}{ point2 } & $x$ & $-8.61496083 e-011$ & $-8.35456843 \mathrm{e}-011$ \\
\hline & $\mathrm{y}$ & $1.78626169 \mathrm{e}-010$ & $1.73227099 \mathrm{e}-010$ & & $\mathrm{y}$ & $1.58897173 \mathrm{e}-010$ & $1.54094418 \mathrm{e}-010$ \\
\hline & $\mathrm{Z}$ & $-9.05761688 \mathrm{e}-009$ & $-8.78384476 \mathrm{e}-009$ & & $\mathrm{z}$ & $-8.56253290 \mathrm{e}-009$ & $-8.30372482 \mathrm{e}-009$ \\
\hline \multirow{3}{*}{ point3 } & $x$ & $-1.49236201 e-009$ & $-1.44725443 e-009$ & \multirow{3}{*}{ point3 } & $x$ & $-1.34730727 \mathrm{e}-009$ & $-1.30658406 \mathrm{e}-009$ \\
\hline & $\mathrm{y}$ & $9.39162498 \mathrm{e}-011$ & $9.10775969 \mathrm{e}-011$ & & $\mathrm{y}$ & $9.65652280 \mathrm{e}-011$ & $9.36465072 \mathrm{e}-011$ \\
\hline & $\mathrm{z}$ & $-6.09558981 e-008$ & $-5.91134679 \mathrm{e}-008$ & & $\mathrm{z}$ & $-7.59917214 \mathrm{e}-008$ & $-7.36948280 \mathrm{e}-008$ \\
\hline
\end{tabular}


Table 8: Displacements of three random points at time $t=0.43 \mathrm{~s}$.

\begin{tabular}{|c|c|c|c|c|c|c|c|}
\hline \multirow{2}{*}{\multicolumn{2}{|c|}{$\frac{\text { space-step }}{\text { time-step }}$}} & \multicolumn{2}{|c|}{ meshes $100 \times 50$} & \multirow{2}{*}{\multicolumn{2}{|c|}{$\frac{\text { space-step }}{\text { time-step }}$}} & \multicolumn{2}{|c|}{ meshes $200 \times 100$} \\
\hline & & $\Delta t=0.01 \mathrm{~s}$ & $\Delta t=0.005 \mathrm{~s}$ & & & $\Delta t=0.01 \mathrm{~s}$ & $\Delta t=0.005 \mathrm{~s}$ \\
\hline \multirow{3}{*}{ point1 } & $x$ & $-4.58530089 \mathrm{e}-011$ & $-4.49015027 \mathrm{e}-011$ & \multirow{3}{*}{ point1 } & $\mathrm{x}$ & $-4.01757308 \mathrm{e}-011$ & $-3.93420331 \mathrm{e}-011$ \\
\hline & $\mathrm{y}$ & $1.30209427 \mathrm{e}-010$ & $1.27507421 \mathrm{e}-010$ & & $\mathrm{y}$ & $1.04505155 \mathrm{e}-010$ & $1.02336549 \mathrm{e}-010$ \\
\hline & $\mathrm{z}$ & $-5.88901372 \mathrm{e}-009$ & $-5.76680970 \mathrm{e}-009$ & & $\mathrm{z}$ & $-4.57663685 e-009$ & $-4.48166615 e-009$ \\
\hline \multirow{3}{*}{ point2 } & $x$ & $-1.98583217 \mathrm{e}-009$ & $-1.94462380 \mathrm{e}-009$ & \multirow{3}{*}{ point2 } & $\bar{x}$ & $-1.00037578 \mathrm{e}-009$ & $-1.00146935 e-009$ \\
\hline & $\mathrm{y}$ & $1.67331357 \mathrm{e}-010$ & $1.63859024 \mathrm{e}-010$ & & $\mathrm{y}$ & $1.82150461 \mathrm{e}-010$ & $1.53282900 \mathrm{e}-010$ \\
\hline & $\mathrm{z}$ & $-8.75875870 \mathrm{e}-008$ & $-8.57700400 \mathrm{e}-008$ & & $\mathrm{z}$ & $-9.16495964 \mathrm{e}-008$ & $-9.25715540 \mathrm{e}-008$ \\
\hline \multirow{3}{*}{ point3 } & $x$ & $-8.68468686 \mathrm{e}-010$ & $-8.50446880 \mathrm{e}-010$ & \multirow{3}{*}{ point3 } & $\bar{x}$ & $-8.20708557 \mathrm{e}-010$ & $-8.30839286 \mathrm{e}-010$ \\
\hline & $\mathrm{y}$ & $4.65154443 e-010$ & $4.55501914 \mathrm{e}-010$ & & $\mathrm{y}$ & $3.23706867 \mathrm{e}-010$ & $3.01363795 \mathrm{e}-010$ \\
\hline & $\mathrm{z}$ & $-7.44696393 e-008$ & $-7.29243084 \mathrm{e}-008$ & & $\mathrm{z}$ & $-7.17228303 e-008$ & $-7.29795175 \mathrm{e}-008$ \\
\hline
\end{tabular}

The consistent shapes in Fig. 12 show that the numerical scheme for time-dependent elliptic membrane shell model with spherical middle surface is stable under the different combinations of time-step and space-step. The consistent displacements in Tables 4-6 indicate the same stability under different time-steps. This is in accordance with the conclusion of our Theorem 5.1, which infers the stability of the adopted numerical scheme. Moreover, the deformations of the middle surface match the change of applied force, which corroborates the efficiency of the model.

\section{Conclusions}

We have discussed theoretical analysis and numerical methods for the time-dependent membrane shell model. First, the existence and uniqueness of the solution of the model were established. Secondly, the spatial variable was discretized by means of a conforming finite element method and the time discretization of the model was performed using the Newmark scheme. Then the existence, uniqueness, stability, convergence and a priori error estimate are discussed. Finally, numerical experiments for a portion of an ellipsoidal shell and a portion of a spherical shell, were presented.

\section{Acknowledgments}

We are very grateful for Professor P.G. Ciarlet's advice and direction. This paper is supported by the National Natural Science Foundation of China (NSFC 11971379, 11571275, 11572244) and by the Natural Science Foundation of Shaanxi Province (2018JM1014).

\section{References}

[1] A. Gilmanov, T. Le and F. Sotiropoulos, A numerical approach for simulating fluid structure interaction of flexible thin shells undergoing arbitrarily large deformations in complex 
domains, J. Comput. Phys., 300(2015), 814-843.

[2] S. Mansoor-Baghaei and A. M. Sadegh, A closed form solution for the impact analysis of elastic ellipsoidal thin shells, T. Wall. Struct., 93(2015), 54-63.

[3] H. Dong, J. Cui, Y. Nie, Q, Ma and Z. Yang, Multiscale computational method for thermoelastic problems of composite materials with orthogonal periodic configurations, Appl. Math. Model, 60(2018), 634-660.

[4] J. Hu and T. Wang, Pre-impact configuration designing of a robot manipulator for impact minimization, J. Mech. Robot., 9(2017):1-10.

[5] S. Erlicher, S. Q. Nguyen and F. Martin, Seismic design by the response spectrum method: A new interpretation of elliptical response envelopes and a novel equivalent static method based on probable linear combinations of modes, Nucl. Eng. Des., 276(2014), 277-294.

[6] X. Cui, S. Li and H. Feng, A triangular prism solid and shell interactive mapping element for electromagnetic sheet metal forming process, J. Comput. Phys., 336(2017), $192-211$.

[7] M. I. Sloika, D. D. Sheka and V. P. Kravchuk: Geometry induced phase transitions in magnetic spherical shell, J. Magn. Magn. Mater., 443(2017), 404-412.

[8] J. Zhang, M. Wang, W. Wang and Y. Zhu, Investigation on egg-shaped pressure hulls, Marine. Structures, 52(2017), 50-66.

[9] P. M. Naghdi, The Theory of Shells and Plates, Springer Berlin Heidelberg, 1973.

[10] P. G. Ciarlet and V. Lods, Asymptotic analysis of linearly elastic shells. II. justification of membrane shell equations, Arch. Rational. Mech. Anal., 136(1996), 119-161.

[11] P. G. Ciarlet and V. Lods, Asymptotic analysis of linearly elastic shells: generalized membrane shells, J. Elasticity, 43(1996),147-188.

[12] C. Mardare, Asymptotic analysis of linearly elastic shells: error estimates in the membrane case, Asymptot. Anal., 17(1998), 31-51.

[13] C. Mardare, The generalized membrane problem for linearly elastic shells with hyperbolic or parabolic middle surface, J. Elasticity, 51(1998), 145-165.

[14] P. G. Ciarlet, Mathematical Elasticity. Vol. III: Theory of Shells. Studies in Mathematics and its Applications, vol. 29. North-Holland publishing Co, Amsterdam, 2000.

[15] P. G. Ciarlet, C. Mardare and X. Shen, Donati compatibility conditions for membrane and flexural shells, Anal. Appl., 13(2015), 685-705.

[16] G. Castiñeira and Á. Rodríguez-Arós, On the justification of viscoelastic elliptic membrane shell equations, J. Elasticity, 130(2018), 85-113.

[17] H. Dong, X. Zheng, J. Cui and Q. Ma, Multi-scale computational method for dynamic thermo-mechanical performance of heterogeneous shell structures with orthogonal periodic configurations, Comput. Methods Appl. Mech. Engrg., 354(2019), 143-180.

[18] X. Shen, J. Jia, S. Zhu, H. Li, L. Bai, T. Wang and X. Cao, The time-dependent generalized membrane shell model and its numerical computation, Comput. Methods Appl. Mech. Engrg., 344(2019), 54-70.

[19] X. Shen and H. Li, The time-dependent Koiter model and its numerical computation, Appl. Math. Model, 55(2018), 131-144.

[20] X. Shen, L. Piersanti and P. Piersanti, Numerical simulations for the dynamics of flexural shells, Math. Mech. Solids, 25(2020), 887-912.

[21] P. G. Ciarlet, Linear and Nonlinear Functional Analysis with Applications. Society for Industrial and Applied Mathematics, Philadelphia, 2013.

[22] P. G. Ciarlet, An Introduction to Differential Geometry with Applications to Elasticity. Springer, Dordrecht, 2005.

[23] P. A. Raviart and J. M. Thomas, Introduction à l'Analyse Numérique des Équations aux 
Dérivées Partielles, Masson, Paris, 1983.

[24] A. Quarteroni and A. Valli, Numerical Approximation of Partial Differential Equations, Springer, Berlin and Heidelberg, 1997.

[25] J. L. Lions and E. Magenes: Non-Homogeneous Boundary Value Problems and Applications, Vol. II. Springer, Berlin and Heidelberg, 1972.

[26] P. G. Ciarlet, The Finite Element Method for Elliptic Problems, vol. 4. North-Holland publishing Co., Amsterdam, 1978.

[27] X. Shen, Q. Yang, L. Li, Z. Gao and T. Wang, Numerical approximation of the dynamic Koiter's model for the hyperbolic parabolic shell, Appl. Numer. Math., 150(2020), 194-205.

[28] F. Hecht, New development in freefem ++, Numer. Math., 20(2012), 251-265.

[29] E. Zampieri and L. F. Pavarino, Explicit second order isogeometric discretizations for acoustic wave problems, Comput. Methods Appl. Mech. Engrg., 348(2019), 776-795.

[30] S. Zhu, L. Dede and A. Quarteroni, Isogeometric analysis and proper orthogonal decomposition for the acoustic wave equation, ESAIM: Math. Model. Numer. Anal., 51(2017),11971221.

[31] T. Fiedler, E. Solorzano and A. Oechsner, Numerical analysis of metallic hollow sphere structures, Mater. Lett., 62(2008), 1204-1207.

[32] J. Ahrens, B. Geveci and C. Law, ParaView: An End-User Tool for Large Data Visualization, Visualization Handbook, Elsevier, 2005. 